\title{
Systems Biology under heat stress in Indian Cattle
}

Raja Ishaq Nabi Khan ${ }_{a \dagger}$, Amit Ranjan Sahu ${ }_{a \dagger}$, Waseem Akram Malla a, Manas Ranjan Praharaj b. Neelima Hosamani $b_{\dagger}$, Shakti Kumar ${ }_{b}$, Smita Gupta $a$, Shweta Sharma a , Archana Saxena a, Anshul Varshney a, Pragya Singh a, Vinay Verma ${ }_{c}$, Puneet Kumar $_{c}$, Gyanendra Singh $c$, Aruna Pandey a, Shikha Saxena a, Ravi Kumar Gandham ${ }_{\mathrm{b} \#}$, Ashok Kumar Tiwari d\#

a Division of Veterinary Biotechnology, Indian Veterinary Research Institute, Bareilly, India b Computational Biology and Genomics, National Institute of Animal Biotechnology, Hyderabad, India

c Division of Physiology and Climatology, Indian Veterinary Research Institute, Bareilly, India ${ }_{\mathrm{d}}$ Division of Biological Standardization, Indian Veterinary Research Institute, Bareilly, India

$\succcurlyeq$ Equal Contributors

\#Corresponding Authors: Ravi Kumar Gandham, ravigandham@niab.org.in Ashok Kumar Tiwari, aktiwari71d@gmail.com

\section{Abstract}

Transcriptome profiling of Vrindavani and Tharparkar cattle revealed that more numbers of genes dysregulated in Vrindavani than in Tharparkar. A contrast in gene expression was observed with $18.5 \%$ of upregulated genes in Vrindavani were downregulated in Tharparkar and $17.5 \%$ upregulated genes in Tharparkar were downregulated in Vrindavani. Functional annotation of genes differentially expressed in Tharparkar and Vrindavani revealed that the systems biology in Tharparkar is moving towards counteracting the effects due to heat stress. Unlike Vrindavani, Tharparkar is not only endowed with higher expression of the scavengers (UBE2G1, UBE2S, and UBE2H) of misfolded proteins but also with protectors (VCP, Serp1, and $C A L R)$ of naïve unfolded proteins. Further, higher expression of the antioxidants in Tharparkar enables it to cope up with higher levels of free radicals generated as a result of heat stress. In this study we found relevant genes dysregulated in Tharparkar in the direction that can counter heat stress.

Keywords: Heat Stress; Transcriptome; Tharparkar; Vrindavani

\section{Introduction}

Cattle being homoeothermic modulate their internal body temperature in sync to environmental temperature by equilibrating the amount of heat produced within the body and dissipating it to the ambient environment. The stress that arises due to disproportionate 
37 thermodynamic behavior between cattle and its surrounding environment is termed as heat stress

381 . Environmental induced hyperthermic stress lowers feed intake, which in turn reduces growth, 39 milk production and reproductive efficiency, thereby negatively affecting the economics of 40 livestock keepers ${ }^{2-4}$. Heat stress has been associated with reduced fertility through its deleterious 41 impact on oocyte maturation and early embryonic development ${ }^{5}$. Increased morbidity and 42 mortality was observed in animals due to immune depressive effect of heat stress ${ }^{6}$.

43 India has a wide variety of indigenous cattle breeds distributed throughout its agro44 climatic zones. These are known for their natural tolerance to tropical heat ${ }^{7,8}$. To meet the 45 growing demand for milk and to combine the heat tolerance and tick resistance of zebu with the 46 productivity of temperate dairy breeds ${ }^{9}$ several crossbreeding programs were taken up in India.

47 Every state had its own crossbreeding policy, which is agro-climatic and breed-specific. Though 48 the zebu crosses with European breeds produced more milk than zebu, they were found not 49 withstanding heat/solar radiation ${ }^{10}$. Crossbreds are susceptible to tropical diseases and require a 50 constant input of good management conditions ${ }^{8}$. Antagonism exists between heat tolerance and 51 milk productivity ${ }^{11}$. The adaptive capacity to heat stress varies between species and genetic 52 groups within species. Among various adaptive mechanisms, physiological adaptability seems to 53 be the primary step in cattle. Sahiwal cows better regulate body temperature in response to heat 54 stress than Karan Fries ${ }^{8}$. It was observed that Ongole cattle rely on the respiration rate to 55 maintain thermal balance, while, Bali cattle rely on rectal temperature ${ }^{12}$. In Brazil, Sindhi and 56 Girolando breeds showed better physiological response to thermal stress than Gir cattle ${ }^{13}$. 57 Increase in respiration rate was reported in Nellore breed when exposed to heat load ${ }^{14}$.

In India, Tharparkar is one among the best dairy breeds. It is adapted to the Indian states 59 of Punjab and Haryana. ${ }^{15,16}$. It is considered to be the hardiest, disease resistant, heat tolerant 60 and tick resistant indigenous cattle breed of the country ${ }^{17}$. This breed has also been used in 61 several crossbreeding programs. Currently, percentage of purebreds is exceptionally low in India 62 (Department of Animal Husbandry \& Dairying, Govt. of India). Most of the farmer's in India 63 have Crossbreds and the percentage of exotic inheritance in these Crossbreds is unknown. 64 Vrindavani, a Crossbred (synthetic), with $27 \%$ of Indigenous blood and $73 \%$ of exotic 65 inheritance ${ }^{18}$ is a representation of the kind of admixture that prevails in Indian cattle. Therefore, 66 comparing Tharparkar with Vrindavani may establish the lost importance of Indigenous cattle 
67 and would further emphasize the need of conserving our indigenous purebreds because of 68 advantageous traits like Heat tolerance.

Studies explaining the difference between the genetic groups (Crossbreds and Indigenous cattle) have been done mainly to address the physiological responses vis - a - vis heat stress and

71 very few studies at the genomic level have been taken up ${ }^{19,20}$.Also, there is an increasing need to 72 develop methods by combining the knowledge from -omics technologies to identify heat tolerant 73 animals ${ }^{11}$. Transcriptome profiling / RNA-Sequencing (RNA-seq) is a high throughput omics 74 approach to measure relative global changes in the transcripts under specific condition(s) ${ }^{21-23}$ to 75 study the systems biology behind a phenotype ${ }^{23,24}$. RNA - seq allows for analysis of 76 transcriptome in an unbiased way, with, a tremendous dynamic detection range $(>8,000$ fold $)$, 77 and low background signals ${ }^{25}$. It has been used as an investigating tool in understanding disease 78 pathogenesis ${ }^{26,27}$ and differential physiological response to various biotic and abiotic factors $79 \quad 28,29$.

In this study, Tharparkar and Vrindavani cattle were subjected to heat stress and blood samples were collected on $0^{\text {th }}$ day and $7^{\text {th }}$ day, as it is known that short term acclimation occurs

82 around 5 - 6 days ${ }^{30,31}$. The transcriptome of $7^{\text {th }}$ day was compared and with $0^{\text {th }}$ day in both the 83 genetic groups to understand their differential response to heat stress.

\section{Results}

\section{Physiological Parameters}

86 The overview of the analysis is given in Figure 1. Respiration rate (RR), rectal 87 temperature $(\mathrm{RT})$ and T3 level increased significantly $(\mathrm{p}<0.05)$ on $7^{\text {th }}-$ day post heat stress in 88 both the genetic groups $(n=5)$ (Figure 2). However, the increase in RR, RT and T3 level, was 89 found significantly $(\mathrm{P}<0.05)$ higher in Vrindavani than in Tharparkar.

\section{Comparison of DEGs of Vrindavani and Tharparkar under heat stress}

91 The differentially expressed genes for each genetic group were obtained on comparing 92 the 0 day and $7^{\text {th }}$ day RNA-seq data using EdgeR after obtaining the gene counts from RSEM. 93 Under heat stress, global expression profiles of Vrindavani and Tharparkar were identified with 946042 and 4718 differentially expressed genes (DEGs), respectively (Supplementary Table 1). 95 Among these, 3481 DEGs were found common between the two genetic groups, while 2561 and 
961238 DEGs were uniquely found in Vrindavani and Tharparkar, respectively (Figure 3a). 97 Additionally, 3132 and 2924 genes were upregulated and downregulated in Vrindavani, 98 respectively, while 2367 and 2358 genes were upregulated and downregulated in Tharparkar, 99 respectively (Figure 3b). On comparison of upregulated and downregulated genes, 724 and 1416 100 genes were found uniquely upregulated and 514 and 1145 genes were found uniquely 101 downregulated in Tharparkar and Vrindavani, respectively. The comparison also revealed that $102 \quad 17.5 \%$ of upregulated genes (1278) in Tharparkar were downregulated in Vrindavani and 18.5\% 103 downregulated genes (1344) in Tharparkar were upregulated in Vrindavani. However, the 104 number of common upregulated and downregulated genes in both the genetic groups were 357 $105(4.9 \%)$ and $498(6.8 \%)$, respectively (Figure 3c).

\section{Functional annotation of DEGs in Reactome}

\section{DEGs in Tharparkar and Vrindavani}

108 On functional annotation of all the DEGs (6042 and 4718 in Vrindavani and Tharparkar, 109 respectively), the commonly enriched significant pathways in both genetic groups included, 110 neutrophil degranulation, L13a-mediated translational silencing of ceruloplasmin expression, 111 Formation of a pool of free 40S subunits, ubiquitination and proteasome degradation, SRP112 dependent cotranslational protein targeting to membrane and nonsense-mediated decay and 113 selenocysteine synthesis. Among the most enriched pathways, peptide chain elongation, gap-

114 filling DNA repair synthesis and ligation in TC-NER were the pathways significantly enriched in 115 Tharparkar and not in Vrindavani. The pathways - SCF-beta-TrCP mediated degradation of 116 Emi1, Autodegradation of Cdh1 by Cdh1:APC/C and Regulation of RAS by GAPs were the 117 pathways significantly enriched in Vrindavani and not in Tharparkar.

\section{Common DEGs in Tharparkar and Vrindavani}

119 On functional annotation of common the DEGs (3481), the enriched significant pathways 120 included, neutrophil degranulation, L13a-mediated translational silencing of ceruloplasmin 121 expression, formation of a pool of free 40S subunits, ubiquitination and proteasome degradation, 122 SRP-dependent cotranslational protein targeting to membrane, RNA Polymerase II Transcription 123 Termination, Peptide chain elongation, nonsense-mediated decay and selenocysteine synthesis.

124 On functionally annotation of 1278 common genes that were upregulated in Tharparkar and 125 downregulated in Vrindavani, the enriched pathways included all the pathways as mentioned for 
126 the common except for ubiquitination \& proteasome degradation. In addition, cellular response

127 to stress was found significantly enriched these genes upregulated in Tharparkar and 128 downregulated in Vrindavani. Further, on assessing the 1344 genes that were upregulated in

129 Vrindavani and downregulated in Tharparkar, RUNX1 regulates transcription of genes involved 130 in BCR signaling, p75NTR recruits signaling complexes, chromatin modifying enzymes,

131 chromatin organization, death receptor signaling, resolution of D-loop structures through

132 Holliday junction intermediates and TP53 regulates transcription of DNA repair genes pathways

133 were significantly enriched.

\section{Unique DEGs in Tharparkar}

135 On functional annotation of unique DEGs (1238) expressed only in Tharparkar, the enriched 136 significant pathways included, nucleotide excision repair, mitotic G1 phase and G1/S transition, 137 DNA replication pre-initiation, cell cycle, synthesis of DNA, translation, DNA repair, activation 138 of the pre-replicative complex, cell cycle checkpoints and inhibition of replication initiation of 139 damaged DNA by RB1/E2F.

\section{Unique DEGs in Vrindavani}

141 On functional annotation of unique DEGs (2561) expressed only in Vrindavani, the enriched 142 significant pathways included, unfolded protein response (UPR), loss of proteins required for 143 interphase microtubule organization from the centrosome, amplification of signal from 144 unattached kinetochores via a MAD2 inhibitory signal, mitotic spindle checkpoint, organelle 145 biogenesis and maintenance and loss of Nlp from mitotic centrosomes.

\section{Functional annotation of DEGs in IPA}

\section{Canonical pathway analysis}

148 Canonical pathway analysis by Ingenuity Pathway Analysis (IPA) revealed contrast in signaling 149 pathways in Vrindavani and Tharparkar. Canonical pathways associated with Vrindavani and 150 Tharparkar are represented in Figure $4 \mathrm{a}$ and $4 \mathrm{~b}$. In Vrindavani, Oncostatin M Signaling, 151 Phospholipase C Signaling, EIF2 Signaling, Integrin Signaling, IL-3 Signaling, and CXCR4 152 Signaling were found to be highly inactivated $(Z-$ score $>2.0)$ and PTEN signaling was found 153 to be highly activated $(Z-$ score < 2.0). In Tharparkar, EIF2 Signaling, Androgen Signaling, 154 Oncostatin M Signaling, $\alpha$-Adrenergic Signaling, BMP signaling pathway, and UVC-Induced 
155 MAPK Signaling were found to be highly activated and PTEN signaling was found to be

156 inactivated. The canonical pathway Oncostatin M Signaling and EIF2 Signaling were found to

157 have the highest ratio of genes involved vis-a-vis the genes in the database in Vrindavani and

158 Tharparkar, respectively.

159 While carrying out comparative analysis through IPA, Calcium-induced T Lymphocyte 160 Apoptosis, BMP signaling pathway, UVC-Induced MAPK Signaling, Regulation of Cellular 161 Mechanics by Calpain Protease, fMLP Signaling in Neutrophils, Melatonin Signaling, and 162 Leukocyte Extravasation Signaling, were found inactivated in Vrindavani and activated in 163 Tharparkar (Supplementary Figure 1). Genes involved in Oncostatin M Signaling- Growth 164 factor receptor-bound protein 2 (GRB2), GTPase HRas (HRAS), Janus kinase 1 (JAK1), Janus 165 kinase 3 (JAK3), Mitogen-activated protein kinase kinase 1 (MAP2K1), Mitogen-activated 166 protein kinase 1 (MAPK1), Oncostatin-M (OSM), Ras-related protein Rap-1b (RAP1B), Ras167 related protein Rap-2a $(R A P 2 A)$, Signal transducer and activator of transcription 1-alpha/beta 168 (STAT1), Signal transducer and activator of transcription 5B (STAT5B), Non-receptor tyrosine169 protein kinase (TYK2), and Ras-related protein (RRAS) were found downregulated in Vrindavani 170 and upregulated in Tharparkar (Figure 5a, b). While the key genes involved in PTEN Signaling 171 pathway - Fas Ligand (FASLG), member of RAS oncogene family (RAP2A), Bcl-2-like protein 17211 (BIM),Caspase-3 (CASP3) and microspherule protein 1 (MSP58) were found upregulated in 173 Vrindavani and downregulated in Tharparkar as well (Figure 6a, b).

174 Variation in microRNAs and Transcription factors

175 IPA, on evaluating the differentially expression genes predicts miRNAs and 176 Transcription Factors (upstream regulators). In Vrindavani, 111 miRNAs were found to be 177 inactivated and 37 activated. In Tharparkar, 205 miRNAs were found to be inactivated and 272 178 activated. Among them, 52 microRNAs were found common between the two genetic groups. 179 Most of the common miRNAs were found activated in Vrindavani and inactivated in Tharparkar 180 (Supplementary Figure 2). miR-4779, miR-4651, miR-1207-5p, miR-6967-5p and miR-504-3p 181 are the top 5 miRNAs that were activated in Vrindavani and inactivated in Tharparkar.

182 Various Transcription factors were found to regulate the expression of the identified 183 DEGs. Transcription factors, 19 in Tharparkar (11 activated and 8 inactivated) and 26 in 184 Vrindavani (8 activated and 18 inactivated) were identified in IPA that regulate the expression of 
DEGs. Among them, PAX5, MTA3, MYC, PROX1 and SMAD7 in Vrindavani and, HMGA1, MAF, MAX NOTCH22 and NCORI in Tharparkar are the top 5 upregulated and activated TFs. On comparing the TFs of Tharparkar and Vrindavani, it was found that BHLHE40, HMGA1, $H M G B 1, I K Z F 1$, and TCF7 were found to be common. BHLHE40, HMGA1, and TCF7 were found to be activated in Tharparkar and inactivated in Vrindavani and it was vice - versa with $H M G B 1$ and IKZF1 (Supplementary Figure 3)

In the diseases and functions category, on evaluating all the DEGs in both the genetic groups, survival of the organism, ubiquitination, ubiquitination of protein and repair of DNA were found activated in Vrindavani and were found either inactivated/ not-activated in Tharparkar. Similarly, homeostasis, development of hematopoietic cells, leukopoiesis was found relatively activated in Tharparkar in comparison to Vrindavani. Further, translation, Expression of protein, translation of protein and degranulation were found inactivated in Vrindavani.

On correlating the results of disease and function with the results of reactome four major processes that were considered to be associated with heat stress viz. elicitation of unfolded protein response (UPR) in cells; Induction of apoptosis; Ubiquitination and; Imbalance in production of ROS and antioxidants. Heat shock genes and its associated genes are involved in elicitation of unfolded protein response (UPR) in cells. Heat shock genes have been found dysregulated under heat stress in both the genetic groups. Most of the genes encoding Heat shock proteins (HSPs) - Heat shock $70 \mathrm{kDa}$ protein 4 (HSPA4), Heat shock cognate $71 \mathrm{kDa}$ protein (HSPB8), Heat shock $70 \mathrm{kDa}$ protein 1A (HSPAlA), Heat shock cognate $71 \mathrm{kDa}$ protein (HSPA8), Heat shock protein HSP 90-beta (HSP9OAB1) and Heat shock protein HSP 90-alpha (HSP90AAl) and heat shock protein regulating factors- Heat shock factor 1 (HSF1) and Eukaryotic Translation Elongation Factor 1 Alpha 1 (EEF1A1) have been found to be downregulated/not-differentially expressed in Vrindavani but upregulated in Tharparkar.

211 in the regulation of expression of heat shock genes was upregulated in Vrindavani and 212 downregulated in Tharparkar.

Among the apoptotic genes, genes encoding Bcl-2-like protein 11 (BCL2L11), Tumor 214 necrosis factor ligand superfamily member 6 (FASLG), TIR domain-containing adapter 
215 molecule 2 (TICAM2), Toll-like receptor 4 (TLR4), Adenomatous polyposis coli protein $216(A P C)$, Caspase-3 (CASP3), Mitogen-activated protein kinase 8 (MAPK8), Mixed lineage 217 kinase domain-like protein $(M L K L)$, Late endosomal/lysosomal adaptor and MAPK and MTOR 218 activator $5(X I P)$, Vimentin (VIM), and High mobility group protein B2 (HMGB2) were found to 219 be upregulated in Vrindavani and downregulated in Tharparkar. The number of upregulated 220 genes involved in achieving the balance of ROS production and antioxidants, were found to be 221 more in Tharparkar than in Vrindavani. Among these, genes encoding Glutathione peroxidase 2223 (GPX3), Nudix Hydrolase 2 (NUDT2), Catalase (CAT), Cytochrome c (CYCS), Copper 223 chaperone for superoxide dismutase (CCS), Peroxiredoxin-5 (PRDX5), Peroxiredoxin-6 224 (PRDX6), Peroxiredoxin-1 (PRDX1), Superoxide dismutase (SOD1), and Cytochrome b-245 225 heavy chain $(C Y B B)$ were found either downregulated/not-differentially expressed in Vrindavani 226 and upregulated in Tharparkar. More number of genes involved in Ubiquitination were 227 differentially expressed in Vrindavani than in the Tharparkar. Genes encoding Ubiquitin228 conjugating enzyme E2 G1 (UBE2G1), Ubiquitin-conjugating enzyme E2 (UBE2S), Ubiquitin229 conjugating enzyme E2 $\mathrm{H}(U B E 2 H)$, Ubiquitin A-52 residue ribosomal protein fusion product 1 230 (UBA52), and Ubiquitin-activating enzyme E1 (UBA1) have been found downregulated/not231 differentially expressed in Vrindavani and upregulated in Tharparkar. However, Valosin232 containing protein (VCP), RING finger protein 40 (RNF40), and Ubiquitin-conjugating enzyme

233 E2 L3 (UBE2L3) have been found downregulated in Vrindavani but not-differentially expressed

234 in Tharparkar. Among the genes involved in Unfolded Protein folding response (UPR), genes 235 encoding Membrane-bound transcription factor site-1 protease (MBTPS1), Cyclic AMP236 responsive element-binding protein 3-like protein 1 (CREB3L1), Stress-associated endoplasmic 237 reticulum protein 1 (SERP1), Glycogen synthase kinase-3 alpha (GSK3A), Eukaryotic 238 translation initiation factor 2 subunit 3 (EIF2S3), Calreticulin (CALR), and Stress-associated 239 endoplasmic reticulum protein 1 (SERP1) have been found downregulated in Vrindavani and 240 upregulated in Tharparkar (Figure 7).

\section{Protein - protein interaction (PPI) network}

242 Among the genes involved in the 4 major processes considered, protein - protein interaction 243 (PPI) network revealed functional importance of HSP70 (HSPA8 and HSPA1A) and ubiquitin 244 (UBB, UBA52), in coordinating genes involved in heat stress. Out of the total 246 genes 
245 identified from the reactome database among these processes, 177 and 194 genes were found to 246 be differentially expressed in Tharparkar and Vrindavani, respectively. Among these 126 genes

247 were found to be commonly differentially expressed in Tharparkar and Vrindavani. PPI network

248 for these common genes between Tharparkar and Vrindavani was constructed (Supplementary

249 Figure 4). In PPI networks, hubs define the functional and structural importance of a network.

250 The genes, which act as hubs in PPI networks were found to be UBB, UBA52, HSPA8, and

251 HSPA1A (Supplementary Figure 4). Among the 4 hubs, UBB was downregulated in both genetic

252 groups and the rest were downregulated in Vrindavani and upregulated in Tharparkar.

253 A change in the expression of the hub protein will have a larger effect than change in 254 expression of non-hub proteins ${ }^{32}$. Therefore, UBB, UBA52, HSPA8, and HSPA1A are taken to 255 be critical for coordinating the changes in systems biology under heat stress. The hubs HSPA8 256 and HSPA1A are connected to genes that are associated with regulation of stress viz. 257 nucleoporins genes - NUP188, NUP155, NUP210 \& NUP214; BAG family molecular chaperone 258 regulators - BAG1, BAG3 \& BAG4; Heat Shock Protein Family A - HSPA5, HSPA4, HSPA12B 259 \& HSPA9; DnaJ Heat Shock Protein Family i.e. HSP40 - DNAJA1, DNAJC2 \& DNAJB6; Heat 260 shock factor - HSF1; Ubiquitin - UBB \& UBA52 and; Sirtuin - SIRT1. The hubs - UBB and 261 UBA52 are connected to molecules of different proteasome subunits viz. ? type subunits 262 PSMA1 \& PSMA2; $\beta$ type subunits - PSMB4 \& PSMB8; ATPase subunits - PSMC2 \& PSMC5 263 and non-ATPase subunits - PSMD2 \& PSMD13. These hubs were also found connected to 264 ubiquitin specific peptidases - USP9X and USP7 and Ubiquitin-conjugating enzyme - UBE2B, 265 UBE2G1, UBE2Z, UBE2H, UBE2J2, UBE2S \& UBE2D2.

\section{Real-time validation}

Six genes (HSF1, SOD1, CALR, GSK3A, CAT \& GPX3) that were upregulated in Tharparkar but downregulated/not expressed in Vrindavani and four genes (CASP3, FASLG, $B C L 2 L 11 \& A P C)$ that were upregulated in Vrindavani but downregulated in Tharparkar were considered for Real time PCR based on their role in heat stress. The expression of genes was in

271 concordance with the RNA- Seq results (Supplementary Figure 5 and Supplementary table 2).

\section{Discussion}

Heat stress is a natural phenomenon that affects domestic animals in tropical, sub-tropical 
275 the summer months combine to make an uncomfortable environment for dairy cattle. Heat stress 276 negatively impacts a variety of dairy parameters resulting in economic losses ${ }^{33}$. Response to 277 heat stress varies with species and genetic groups within species 5,34,35. In this study, 278 transcriptome of genetic groups - Vrindavani and Tharparkar cattle under heat stress was 279 evaluated to understand their differential response to heat stress.

280 Animals $(\mathrm{n}=5)$ of both the genetic groups were exposed to a temperature of $42{ }^{\circ} \mathrm{C}$ for 7 281 days. Around $5^{\text {th }}-6^{\text {th }}$ day, short term heat acclimation occurs ${ }^{30,31}$. This time point was selected 282 to understand the differences in systems biology to heat stress in the two genetic groups. 283 Initially, heat stress indicators - RR, RT, and T3 level were evaluated. RR was found to increase 284 in both genetic groups under heat treatment and the increase in Vrindavani was found to be 285 significantly $(\mathrm{P}<0.05)$ different from that in Tharparkar. A positive correlation exists between 286 RR and heat treatment ${ }^{36-38}$. This increase is an attempt to dissipate excess body heat by 287 vaporizing more moisture in expired air or response to a greater requirement of oxygen by 288 tissues under heat stress. Also, the physiological response to heat stress includes reduced heat 289 production, which is achieved by lowering feed intake and thyroid hormone secretion ${ }^{39}$. T3 level 290 increases under heat stress ${ }^{40,41}$. A significant increase in T3 level in Vrindavani as compared to 291 Tharparkar indicates an effective regulatory mechanism in modulating T3 levels in Tharparkar in 292 response to heat stress. The T3 triggered metabolism may be one of the reasons that increases 293 heat production resulting in high rectal temperature in Vrindavani in comparison to Tharparkar 294 as was found in our study. The significant increase in RR, RT and T3 level in Crossbreed than in 295 Tharparkar, suggests the inability of Vrindavani to cope up with heat stress in comparison to 296 Tharparkar.

297 A phenotype is defined by the changes in systems biology. Transcriptome profiling by 298 RNA-seq is the most common methodology to study the changes in systems biology. RNA 299 profiling based on next-generation sequencing enables to measure and compare gene expression 300 patterns $^{21}$. The transcriptome of Tharparkar and Vrindavani indicated differential response to 301 heat stress as evident from the DEGs, which were either distinct to both or have a difference in 302 expression. The number of DEGs were higher in Vrindavani than in Tharparkar, suggesting a 303 greater dysregulation in systems biology in Vrindavani. Among the dysregulated genes, the 304 number of upregulated genes were more than the downregulated genes in both genetic groups. 305 However, a contrast in expression was observed with $18.5 \%$ of upregulated genes in Vrindavani, 
306 were downregulated in Tharparkar and $17.5 \%$ upregulated genes in Tharparkar were 307 downregulated in Vrindavani.

The differentially expressed genes in each genetic group were functionally annotated

309 using both reactome and IPA. In the reactome, the enriched pathways were identified and in IPA,

310 based on the expression of the DEGs the activated/inactivated/not-activated pathways were

311 identified. In reactome, on functionally annotating the DEGs of each of the genetic groups, key

312 pathways related to stress - neutrophil degranulation ${ }^{42}$, L13a-mediated translational silencing of

313 ceruloplasmin expression ${ }^{43}$, ubiquitination and proteasome degradation ${ }^{44}$, SRP-dependent

314 cotranslational protein targeting to membrane ${ }^{45}$, Nonsense-Mediated Decay ${ }^{46}$ and

315 selenocysteine synthesis ${ }^{47}$ were enriched in both the genetic groups. Among the common genes,

316 the genes upregulated in Tharparkar but downregulated in Vrindavani showed enrichment

317 towards cellular response to stress besides the above-mentioned pathways. This indicated that

318 Tharparkar must be responding to withstand heat stress. Further, on evaluating the common

319 genes that were upregulated in Vrindavani and downregulated in Tharparkar, pathways -

320 chromatin modifying enzymes, chromatin organization and death receptor signaling indicated

321 towards apoptosis and DNA repair in Vrindavani. These findings were further corroborated with

322 the pathway enrichment analysis of unique genes in both the genetic groups. IPA analysis of

323 disease and biological function showed repair of DNA and ubiquitination, activated in

324 Vrindavani and senescence and degranulation of cells inactivated in Tharparkar. The results from

325 reactome and IPA suggested Tharparkar may be more resilient than Vrindavani.

326 IPA revealed activation or inactivation of several pathways in both the genetic groups. It

327 is known that - EIF2 signalling, helps in initiation of global protein translation ${ }^{48}$; MAPK-

328 signalling pathway, induces cell proliferation ${ }^{49}$; androgen signalling, enhances pro-survival and

329 anti-apoptotic activity in cell ${ }^{50}$; a-Adrenergic signalling, maintains immune defence mechanism

$330{ }^{51}$ and, helps in tissue repair upon stress ${ }^{52}$ and increases angiogenesis ${ }^{53}$; integrin pathway, resists

331 the cell against apoptosis and other environmental insults ${ }^{54}$; IL-3 signalling, aids in cell survival

332 and haematopoiesis ${ }^{55}$; CXCR4 signalling modulates cell survival and cell motility ${ }^{56}$ and ;

333 Phospholipase $\mathrm{C}$ signalling aids in cell survival in stress through protein kinase $\mathrm{C}$ dependent

334 phosphorylation of BCL-2 ${ }^{57}$. Inactivation of these pathways except MAPK-signalling pathway

335 in Vrindavani and activation of $\alpha$-Adrenergic signalling, Androgen signalling, EIF2 signalling 
and MAPK signalling in Tharparkar indicates that the systems biology in Tharparkar is moving towards countering the effects due to heat stress.

On correlating the reactome data with the IPA four major physiological processes elicitation of unfolded protein response (UPR) in cells; Ubiquitination; Induction of apoptosis and; Imbalance in production of ROS and antioxidants were considered for further evaluation

341 (Figure 8). Heat shock and its associated genes are involved in elicitation of unfolded protein 342 response (UPR) in cells. Most of the heat shock genes were found upregulated in Tharparkar and 343 downregulated in Vrindavani. The increased HSP levels have been found positively correlated 344 with tolerance in many species ${ }^{58,59}$. HSF1, that positively regulates the transcription of HSP70 345 and HSP90 60,61 was found upregulated in Tharparkar and downregulated in Vrindavani. 346 Upregulation of HSF1, HSP70 and HSP9O in Tharparkar and vice-versa in Vrindavani 347 corroborates to state that Tharparkar is better equipped to counter heat stress than Vrindavani. 348 Further, to ensure that the HSP70 in Tharparkar is maintained at an optimum level, dysregulation 349 of $C A M K 2 D$ and $G S K 3 A$ seems to act as negative feedback. CAMK2D that induces the 350 transcription of HSP70 via HSF1 ${ }^{62}$ was found downregulated in Tharparkar. GSK3A that 351 inhibits the trimerization of HSF1 that is needed for the induction of HSP70 ${ }^{63}$ was found 352 upregulated in Tharparkar. The decreased level of HSP70 in Vrindavani makes it inevitable that 353 such negative feedbacks would further reduce its level and hence, GSK3A was found 354 downregulated and CAMK, upregulated (Figure 8).

355 Ubiquitination is positively correlated with heat tolerance ${ }^{64,65}$. Ubiquitin-Proteasome 356 System (UPS) regulates the levels of proteins and acts by removing the misfolded or damaged 357 proteins that may accumulate as a result of exposure to abiotic stress. Malfunctioning of 358 ubiquitin-proteasome system (UPS) could have negative consequences for protein regulation, 359 including loss of function ${ }^{66}$. In Tharparkar after heat acclimation, HSP70 tends to activate the 360 ubiquitination pathway to minimize the accumulation of the unfolded proteins that can't be 361 refolded by it ${ }^{67}$. This pathway activation is supported by upregulation of E2 ligases - UBE2G1, $362 U B E 2 S$, and $U B E 2 H$ that catalyze covalent attachment of E2 to E3 ${ }^{68-71}$ in Tharparkar. USP7 363 that deubiquitinates target proteins ${ }^{72,73}$ was found upregulated in Vrindavani and downregulated 364 in Tharparkar. Further, a group of molecules - VCP, SERP1, and CALR that ensure the 365 protection of naïve proteins during their transport within the cell ${ }^{74-76}$ were found upregulated in 
Tharparkar and downregulated in Vrindavani. Unlike Vrindavani, Tharparkar is not only endowed with higher expression of the scavengers of misfolded proteins but also with protectors of naïve unfolded proteins.

Activation of apoptosis pathway is one of the major physiological processes linked with heat stress. Among the apoptotic genes, BCL2L11, FASLG, MLKL, CASP3, MAPK8, and VIM have been found upregulated in Vrindavani and downregulated in Tharparkar under heat stress.

372 BCL2L11 induces apoptosis by neutralizing key molecules of pro-survival BCL2 sub-family 77,78 , FASLG transduces the apoptotic signal into cells ${ }^{79,80}$, CASP3 activates caspases and 374 executes apoptosis ${ }^{81}$, and MAPK8, MLKL, and VIM also induce apoptosis ${ }^{82,83}$. PTEN 375 signaling pathway that drives apoptosis ${ }^{84,85}$ was found inactivated in Tharparkar and activated in

376 Vrindavani. This indicates a relatively higher probability of apoptosis in Vrindavani than in 377 Tharparkar.

The ability to balance the ROS and antioxidant level, is one of the key factors that would determine the tolerance of an individual to heat stress. The antioxidant triad of GPX, SOD, and

380 CAT that forms the first line of defense against reactive oxygen species ${ }^{86-88}$, was found 381 upregulated in Tharparkar and downregulated in Vrindavani. Additionally, genes belonging to 382 Peroxiredoxins - PRDX3, PRDX5 and PRDX6 that catalyze the reduction of hydrogen peroxide 383 and organic hydroperoxides ${ }^{89-93}$,were also found upregulated in Tharparkar and were either 384 downregulated or not-differentially expressed in Vrindavani. Higher expression of the 385 antioxidants in Tharparkar enables it to cope up with higher levels of free radicals generated as a 386 result of heat stress while Vrindavani is unable to do so.

\section{Conclusion}

388 A contrast in expression was observed with $18.5 \%$ of upregulated genes in Vrindavani 389 were downregulated in Tharparkar and $17.5 \%$ upregulated genes in Tharparkar were 390 downregulated in Vrindavani. Transcripts of molecules that stimulate heat shock response, 391 Ubiquitination, unfolded protein response and antioxidant level were found upregulated in 392 Tharparkar and downregulated in Vrindavani. EIF2 Signaling that promotes protein translation 393 and PTEN signaling that drives apoptosis were found activated and inactivated in Tharparkar, 394 respectively and vice-versa in Vrindavani. We found relevant molecules/genes dysregulated in 395 Tharparkar in the direction that counters heat stress. A proposed contrasting interplay of 
molecules in both the two groups is shown in Figure 8. To the best of our knowledge this is a comprehensive comparison between Tharparkar and Vrindavani at a global level using transcriptome analysis.

\section{Methods}

\section{Experimental condition and Ethical Statement}

The animals used for the study were from the Indian Veterinary Research Institute. The permission to conduct the study was granted by Indian Veterinary Research Institutional Animal

403 Ethics Committee (IVRI-IAEC) under the Committee for Control and Supervision of 404 Experiments on Animals (CPCSEA), India, vide letter no 387/CPSCEA. Genetic groups 405 Tharparkar (Indigenous breeds) and Vrindavani (synthetic Crossbred) were considered in this 406 study. Prior to experiment, the animals - 05 Tharparkar and 05 Vrindavani cattle, were 407 acclimatized for 15 days outside the Psychometric chamber. The experiment was conducted 408 during October when the environmental Temperature Humidity Index (THI) was 73.0242. These 409 animals were exposed in Psychometric chamber at $42{ }^{\circ} \mathrm{C}$ for six hours for 7 days (THI 410 =78.5489). All the animals were fed with wheat straw and concentrate mixture in 60:40 ratios. 411 Respiration rate (RR) and rectal temperature (RT) of animals from each genetic group were 412 measured on 0 day (Control, $n=5$ ) before exposure to Psychometric chamber and on $7^{\text {th }}$ day of 413 heat exposure (Treated, $n=5$ ). Blood samples were collected from the animals at the mentioned 414 time points and serum concentration of Triiodothyronine (T3) was estimated by RIA technique 415 using $\mathrm{T}_{3}{ }^{125} \mathrm{I}$ (Immunotech) as per the manufacturer's instructions.

416 RNA sequencing (RNA-seq)

417 PBMCs were collected from the blood samples using Ficol histopaque gradient method. 418 Total RNA from each of the collected samples (PBMCs) was isolated using the RNeasy Mini kit 419 (Qiagen GmbH, Germany) according to the manufacturer's protocol. The integrity and quantity 420 of isolated RNA were assessed on a Bioanalyzer 2100 (Agilent Technologies, Inc). The library 421 was prepared using NEBNext Ultra RNA Library Prep Kit for Illumina (NewEngland Biolabs 422 Inc.) following the manufacturer's protocol. Approximately, 100ng of RNA from each sample 423 was used for RNA library preparation. The quality of the libraries was assessed on Bioanalyzer. 424 Libraries were quantified using a Qubit 2.0 Fluorometer (Life technologies) and by qPCR. 425 Library $(1.3 \mathrm{ml}, 1.8 \mathrm{pM})$ was denatured, diluted and loaded onto a flow cell for sequencing. 
426 cDNA library preparation and Illumina Sequencing was performed at Sandor Life Sciences Pvt.

427 (Hyderabad, India). Finally, the RNA-seq data were provided in FASTQ format.

\section{Data processing}

The sequenced reads were paired-end and 150bp in length. Quality control checks on raw sequence data from each sample were performed using FastQC (Babraham Bioinformatics).

431 Processing of the data was performed using prinseq-lite software ${ }^{94}$ to remove reads of low 432 quality (mean phred score 25) and short length $(<50)$ for downstream analysis. The data was 433 submitted to the GEO database with accession number GSE136652.

\section{Identification of Differentially Expressed Genes (DEGs)}

Bos taurus reference genome (release 94) and its associated gene transfer file (GTF) were downloaded from Ensembl FTP genome browser ${ }^{95}$. The reference genome was prepared and 437 indexed by RNA-Seq by expectation maximization (RSEM) ${ }^{96}$ by rsem-prepare-reference 438 command. Further, the clean reads obtained from filtering of raw data were aligned to the 439 indexed reference genome by Bowtie ${ }^{97}$ to estimate gene abundance in counts by rsem440 calculate-expression command. To compare the gene expression levels among different samples, 441 the aligned reads were used to generate a data matrix by rsem-generate-data-matrix command. 442 In each genetic group, all the samples of day 7 (treated) were compared with the day 0 (Control) 443 for the calculation of differential gene expression by edgeR ${ }^{98}$ package. The Ensemble IDs of the 444 differentially expressed genes (DEGs) were converted to the respective gene ID by g: Convert of 445 g: Profiler ${ }^{99,100}$.

\section{Functional Analysis of DEGs - Reactome and Ingenuity pathway analysis (IPA)}

447 The differentially expressed genes in both the genetic groups were functionally annotated 448 in Reactome ${ }^{101}$ and IPA (Ingenuity Pathway Analysis). Reactome provides bioinformatics tools 449 for visualisation, interpretation and analysis of pathway knowledge to support basic research. 450 Reactome analysis was done for all DEGs in both genetic groups, common DEGs and for unique 451 DEGs for each genetic group. For common DEGs, analysis was also done for contrasting genes 452 (genes upregulated in Tharparkar but downregulated in Vrindavani and the vice-versa). 
455 placing experimental results within the context of biological systems. Here, IPA was used to 456 analyze the identified DEGs of Vrindavani and Tharparkar. The list of DEGs from each genetic 457 group was used to identify the canonical pathways and the most significant biological processes 458 against Ingenuity Pathways Knowledge Base (IKB). Core analysis for each dataset was 459 performed to know activated $(\mathrm{Z}$ score $>2)$ or inactivated $(\mathrm{Z}$ score < -2$)$ canonical pathways. 460 Upstream regulators - Transcription factors and microRNAs were identified. Significant 461 Diseases and functions were also identified.

462 The results obtained from Reactome and IPA were correlated to narrow down to 463 processes - Induction of apoptosis, Ubiquitination, elicitation of unfolded protein response 464 (UPR) in cells and Imbalance in production of ROS and antioxidants. The unique genes involved 465 in all related pathways to the processes mentioned above were extracted from the Reactome 466 analysis and were found to be 246 in number. From among these genes the number of 467 differentially expressed genes in Tharparkar and Vrindavani, and the common genes between the 468 genetic groups were extracted.

\section{Predicted protein-protein interaction network}

470 Protein interaction network (interactome) analysis provides an effective way to 471 understand the interrelationships between genes ${ }^{103}$. Protein-protein interactions (PPI) among the 472 common genes involved in the processes mentioned, were retrieved using String database ${ }^{104}$. 473 The degree was calculated using igraph package (https://cran.r-project.org/web/packages/igraph/ 474 index.html). The PPI network was then visualized using Cytoscape software V. $3.7^{105}$ using 475 DEGs and degree value from igraph.

\section{Validation of reference genes identified}

477 The quantity of data generated from RNA sequencing is large and therefore it is 478 important to validate differential expression by real-time RT-PCR. Genes - BCL2L11, FASLG, 479 CASP3, CAT, SOD1, GSK3A, CALR, HSF1, APC, and GPX3 were selected based on their role in 480 heat stress and qRT-PCR was performed on Applied Biosystems 7500 Fast system. GAPDH was 481 taken as the internal control. Each of the samples was run in triplicates and relative expression of 482 each gene was calculated using the $2^{-\Delta \Delta C T}$ method with control as the calibrator ${ }^{106}$.

\section{Statistical Analysis}


Respiration rate, Rectal temperature and T3 level were compared using student's $t$-test in

485 JMP9 (SAS Institute Inc., Cary, USA) to test the significance of the difference between the

486 control ( 0 day) and treated $\left(7^{\text {th }}\right.$ day). This comparison was done within and between genetic

487 groups. Differences within/between groups were considered significant at $P \leq 0.05$.

\section{Declarations}

\section{Ethics approval and consent to participate}

490 The permission to conduct the study was granted by Indian Veterinary Research Institutional

491 Animal Ethics Committee (IVRI-IAEC) under the Committee for Control and Supervision of

492 Experiments on Animals (CPCSEA), India, vide letter no 387/CPSCEA.

\section{Consent for publication}

494 Not applicable.

\section{Availability of data and materials}

496 The data was submitted to the GEO database with accession number GSE136652

\section{Competing interests}

498 None of the authors had a conflict of interest to declare

\section{$499 \quad$ Funding}

500 This study was supported by "National Innovations in Climate Resilient Agriculture (NICRA) -

501 Identification of unique factors in indigenous livestock making them resilient to climate change

502 in relation to diseases" for the funds to carryout Sampling, Wet Lab experiments and for the 503 procurement of License of IPA.

\section{Authors' contributions}

505 AKT and RG conceived and designed the research. SG, SmS, AS,AV, VV, PK, ShS and GS

506 conducted the wet lab work. RINK, ARS, NH, WAM, MRP, SK, AP and RG analyzed the data. 
RINK, ARS, MRP, RG, AS and GS helped in manuscript drafting and editing. AKT and RG

508 proofread the manuscript

\section{Acknowledgements}

510 We also thank Department of Biotechnology, Govt of India for providing fellowship and

511 contingency for RK (DBT Fellow No. DBT/2017/IVRI/768), ARS (DBT Fellow No.

512 DBT/2014/IVRI/170) and WAM (DBT Fellow No. DBT/2017/IVRI/769).

\section{References}

5141 Mehla, K. et al. Genome-wide analysis of the heat stress response in Zebu (Sahiwal) cattle. Gene 533, 500-507 (2014). Armstrong, D. V. Heat stress interaction with shade and cooling. J. Dairy Sci. 77, 2044-2050, doi:10.3168/jds.S0022-0302(94)77149-6 (1994).

3 Rojas-Downing, M. M., Nejadhashemi, A. P., Harrigan, T. \& Woznicki, S. A. Climate change and

4 Hahn, G. Dynamic responses of cattle to thermal heat loads. J. Anim. Sci. 77, 10-20 (1999).

5 Dash, S. et al. Effect of heat stress on reproductive performances of dairy cattle and buffaloes: A review. Veterinary world 9, 235 (2016).

6 Renaudeau, D. et al. Adaptation to hot climate and strategies to alleviate heat stress in livestock production. Animal 6, 707-728 (2012). Berman, A. Invited review: Are adaptations present to support dairy cattle productivity in warm 7 Berman, A. Invited review: Are adaptations

8 Rashamol, V. P. et al. Physiological adaptability of livestock to heat stress: an updated review. $J$ Anim Behav Biometeorol 6, 62-71 (2018).

9 Davis, S. R., Spelman, R. J. \& Littlejohn, M. D. BREEDING AND GENETICS SYMPOSIUM:Breeding heat tolerant dairy cattle: the case for introgression of the "slick" prolactin receptor variant into dairy breeds. J Anim Sci 95, 1788-1800, doi:10.2527/jas.2016.0956 (2017).

10 Mcmanus, C. M., Louvandini, H., Paim, T. P., Silva, F. C. P. e. \& Bernal, F. E. M. Factors affecting heat tolerance in crossbred cattle in central Brazil. Ciência Animal Brasileira 15, 152-158 (2014).

11 Carabano, M. J., Ramon, M., Menendez-Buxadera, A., Molina, A. \& Diaz, C. Selecting for heat tolerance. Anim Front 9, 62-68, doi:10.1093/af/vfy033 (2019).

12 Aritonang, S., Yuniati, R., Abinawanto, Imron, M. \& Bowolaksono, A. in AIP Conference Proceedings. 030098 (AIP Publishing).

13 Cardoso, C. et al. Physiological and thermographic response to heat stress in zebu cattle. Livestock Science 182, 83-92 (2015). subjected to heat stress. Semina: Ciências Agrárias 36, 4565-4574 (2015). Sodhi, M., Mukesh, M., Prakash, B., Ahlawat, S. \& Sobti, R. Microsatellite DNA typing for assessment of genetic variability in Tharparkar breed of Indian zebu (Bos indicus) cattle, a major breed of Rajasthan. Journal of genetics 85, 165-170 (2006). 
Choudhary, S. et al. Tharparkar: The pride of desert. Journal of entomology and zoology studies 6, 1915-1919 (2018). Ahmad, S. F. et al. Revelation of genomic breed composition in a crossbred cattle of India with the help of Bovine50K BeadChip. Genomics 112, 1531-1535, doi:10.1016/j.ygeno.2019.08.025 (2020).

Salleh, M. et al. RNA-Seq transcriptomics and pathway analyses reveal potential regulatory genes and molecular mechanisms in high-and low-residual feed intake in Nordic dairy cattle. BMC Genomics 18, 258 (2017). bovine milk transcriptome using RNA-Seq technology. Mamm. Genome 21, 592-598 (2010).

21 Mortazavi, A., Williams, B. A., McCue, K., Schaeffer, L. \& Wold, B. Mapping and quantifying mammalian transcriptomes by RNA-Seq. Nat. Methods 5, 621 (2008). Wang, Z., Gerstein, M. \& Snyder, M. RNA-Seq: a revolutionary tool for transcriptomics. Nature reviews genetics 10, 57 (2009). Peste-des-petits-ruminants virus infected goats. Front. Immunol. 10 (2019). Manjunath, S. et al. Comparative and temporal transcriptome analysis of peste des petits ruminants virus infected goat peripheral blood mononuclear cells. Virus Res. 229, 28-40 (2017). Li, P., Piao, Y., Shon, H. S. \& Ryu, K. H. Comparing the normalization methods for the differential analysis of Illumina high-throughput RNA-Seq data. BMC Bioinformatics 16, 347 (2015). Khanduri, A. et al. Dysregulated miRNAome and proteome of PPRV infected goat PBMCs reveal a coordinated immune response. Front. Immunol. 9, 2631 (2018).

27 Pandey, A. et al. Modulation of host miRNAs transcriptome in lung and spleen of Peste des Petits ruminants virus infected sheep and goats. Front. Microbiol. 8, 1146 (2017). bovine leukocytes using RNA-seq technology. BMC Genomics 13, 250 (2012). 73-79 (2014). stress acclimation in goat (Capra hircus). Gene 575, 543-550 (2016).

31 Garrett, A. et al. Short-term heat acclimation is effective and may be enhanced rather than impaired by dehydration. Am. J. Hum. Biol. 26, 311-320 (2014).

$32 \mathrm{He}, \mathrm{X}$. \& Zhang, J. Why do hubs tend to be essential in protein networks? PLoS Genet 2, e88, doi:10.1371/journal.pgen.0020088 (2006).

33 Polsky, L. \& von Keyserlingk, M. A. Invited review: Effects of heat stress on dairy cattle welfare. J. Dairy Sci. 100, 8645-8657 (2017).

34 Ross, J. et al. Physiological consequences of heat stress in pigs. Animal Production Science 55, 1381-1390 (2015).

35 Mayorga, E. J., Renaudeau, D., Ramirez, B. C., Ross, J. W. \& Baumgard, L. H. Heat stress adaptations in pigs. Animal Frontiers 9, 54-61 (2018).

36 Blackshaw, J. K. \& Blackshaw, A. Heat stress in cattle and the effect of shade on production and behaviour: a review. Australian Journal of Experimental Agriculture 34, 285-295 (1994).

37 Beede, D. \& Collier, R. Potential nutritional strategies for intensively managed cattle during thermal stress. J. Anim. Sci. 62, 543-554 (1986).

38 Ganaie, A. et al. Biochemical and physiological changes during thermal stress in bovines: A review. Iranian Journal of Applied Animal Science 3, 423-430 (2013).

594 Sci. 82, 349-360 (2004). 
59540 Magdub, A., Johnson, H. \& Belyea, R. Effect of environmental heat and dietary fiber on thyroid physiology of lactating cows. J. Dairy Sci. 65, 2323-2331 (1982).

41 Williamson, R., Misson, B. \& Davison, T. The effect of exposure to 40 on the heat production and the serum concentrations of triiodothyronine, thyroxine, and corticosterone in immature domestic fowl. Gen. Comp. Endocrinol. 60, 178-186 (1985).

60042 Rodeberg, D. A., Meyer, J. G. \& Babcock, G. F. Heat shock response: presence and effects in burn patient neutrophils. Journal of leukocyte biology 66, 773-780, doi:10.1002/jlb.66.5.773 (1999). Orzheshkovskyi, V. V. \& Trishchynska, M. A. Ceruloplasmin: Its Role in the Physiological and Pathological Processes. Neurophysiology 51, 141-149, doi:10.1007/s11062-019-09805-9 (2019). Flick, K. \& Kaiser, P. Protein degradation and the stress response. Seminars in cell \& developmental biology 23, 515-522, doi:10.1016/j.semcdb.2012.01.019 (2012). Zhang, D., Sweredoski, M. J., Graham, R. L., Hess, S. \& Shan, S. O. Novel proteomic tools reveal essential roles of SRP and importance of proper membrane protein biogenesis. Molecular \& cellular proteomics : MCP 11, M111 011585, doi:10.1074/mcp.M111.011585 (2012). Goetz, A. E. \& Wilkinson, M. Stress and the nonsense-mediated RNA decay pathway. Cellular and molecular life sciences : CMLS 74, 3509-3531, doi:10.1007/s00018-017-2537-6 (2017). Hariharan, S. \& Dharmaraj, S. Selenium and selenoproteins: it's role in regulation of inflammation. Inflammopharmacology 28, 667-695, doi:10.1007/s10787-020-00690-x (2020). Rajesh, K. et al. Phosphorylation of the translation initiation factor elF2alpha at serine 51 determines the cell fate decisions of Akt in response to oxidative stress. Cell Death Dis 6, e1591, doi:10.1038/cddis.2014.554 (2015).

Zhang, W. \& Liu, H. T. MAPK signal pathways in the regulation of cell proliferation in mammalian cells. Cell Res 12, 9-18, doi:10.1038/sj.cr.7290105 (2002).

Shiota, M., Yokomizo, A. \& Naito, S. Pro-survival and anti-apoptotic properties of androgen receptor signaling by oxidative stress promote treatment resistance in prostate cancer. Endocr Relat Cancer 19, R243-253, doi:10.1530/ERC-12-0232 (2012).

51 Lacoste, A., De Cian, M. C., Cueff, A. \& Poulet, S. A. Noradrenaline and alpha-adrenergic signaling induce the hsp70 gene promoter in mollusc immune cells. J Cell Sci 114, 3557-3564 (2001).

52 Xian, D. et al. Emerging Roles of Redox-Mediated Angiogenesis and Oxidative Stress in Dermatoses. Oxid Med Cell Longev 2019, 2304018, doi:10.1155/2019/2304018 (2019).

53 Zhao, X. et al. Overexpression of Cardiomyocyte alpha1A-Adrenergic Receptors Attenuates Postinfarct Remodeling by Inducing Angiogenesis Through Heterocellular Signaling. Arterioscler Thromb Vasc Biol 35, 2451-2459, doi:10.1161/ATVBAHA.115.305919 (2015). Stupack, D. G. \& Cheresh, D. A. Get a ligand, get a life: integrins, signaling and cell survival. J Cell Sci 115, 3729-3738, doi:10.1242/jcs.00071 (2002). Reddy, E. P., Korapati, A., Chaturvedi, P. \& Rane, S. IL-3 signaling and the role of Src kinases, JAKs and STATs: a covert liaison unveiled. Oncogene 19, 2532-2547, doi:10.1038/sj.onc.1203594 (2000). Regulation of CXCR4-Mediated Signaling in Prostate Cancer Cells is Dependent on Cellular Oxidative State. Cell Physiol Biochem 37, 2071-2084, doi:10.1159/000438566 (2015).

57 Bai, X. C. et al. Phospholipase C-gamma1 is required for survival in heat stress: involvement of protein kinase C-dependent $\mathrm{Bcl}-2$ phosphorylation. J Biochem 131, 207-212, doi:10.1093/oxfordjournals.jbchem.a003089 (2002).

58 Parsell, D. \& Lindquist, S. The function of heat-shock proteins in stress tolerance: degradation and reactivation of damaged proteins. Annu. Rev. Genet. 27, 437-496 (1993). 

70 gene in tissues and heat stress phenotypes in goats during peak heat stress period. Cell Stress Chaperones 21, 645-651 (2016). Westerheide, S. D., Anckar, J., Stevens, S. M., Sistonen, L. \& Morimoto, R. I. Stress-inducible regulation of heat shock factor 1 by the deacetylase SIRT1. Science 323, 1063-1066 (2009). Prodromou, C. Mechanisms of Hsp90 regulation. Biochem. J. 473, 2439-2452 (2016). Holmberg, C. I. et al. Phosphorylation of serine 230 promotes inducible transcriptional activity of heat shock factor 1 . The EMBO journal 20, 3800-3810 (2001). chaperone gene transcription involves the serine phosphorylation, $14-3-3 \varepsilon$ binding, and cytoplasmic sequestration of heat shock factor 1. Mol. Cell. Biol. 23, 6013-6026 (2003).

653 Ryu, K. Y. et al. The mouse polyubiquitin gene UbC is essential for fetal liver development, cell-cycle progression and stress tolerance. The EMBO journal 26, 2693-2706 (2007). Lyzenga, W. J. \& Stone, S. L. Abiotic stress tolerance mediated by protein ubiquitination. J. Exp. Bot. 63, 599-616 (2011). Sulistio, Y. A. \& Heese, K. The ubiquitin-proteasome system and molecular chaperone deregulation in Alzheimer's disease. Mol. Neurobiol. 53, 905-931 (2016). Fernández-Fernández, M. R., Gragera, M., Ochoa-Ibarrola, L., Quintana-Gallardo, L. \& Valpuesta, J. M. Hsp70-a master regulator in protein degradation. FEBS Lett. 591, 2648-2660 (2017). Pabarcus, M. K. et al. CYP3A4 ubiquitination by gp78 (the tumor autocrine motility factor receptor, AMFR) and CHIP E3 ligases. Arch. Biochem. Biophys. 483, 66-74 (2009). polyubiquitination to preferred lysines. J. Biol. Chem. 285, 8595-8604 (2010). Sheng, Y. et al. A human ubiquitin conjugating enzyme (E2)-HECT E3 ligase structure-function screen. Mol. Cell. Proteomics 11, 329-341 (2012). Kaiser, P. et al. A human ubiquitin-conjugating enzyme homologous to yeast UBC8. J. Biol. Chem. 269, 8797-8802 (1994). $\mathrm{Li}, \mathrm{M}$. et al. Deubiquitination of p53 by HAUSP is an important pathway for p53 stabilization. Nature 416, 648 (2002). Zhao, Y. et al. Noncanonical regulation of alkylation damage resistance by the OTUD4 deubiquitinase. The EMBO journal 34, 1687-1703 (2015). Kadowaki, H. et al. Pre-emptive quality control protects the ER from protein overload via the proximity of ERAD components and SRP. Cell Rep. 13, 944-956 (2015). translocation sites, leads to ER stress. Mol. Cell. Biol. 26, 4257-4267 (2006).

in the biosynthesis of myeloperoxidase. J. Biol. Chem. 270, 4741-4747 (1995).

77 O'Connor, L. et al. Bim: a novel member of the Bcl-2 family that promotes apoptosis. The EMBO journal 17, 384-395 (1998).

78 Marani, M., Tenev, T., Hancock, D., Downward, J. \& Lemoine, N. R. Identification of novel isoforms of the BH3 domain protein Bim which directly activate Bax to trigger apoptosis. Mol. Cell. Biol. 22, 3577-3589 (2002).

79 Muneta, Y., Shimoji, Y., Inumaru, S. \& Mori, Y. Molecular cloning, characterization, and expression of porcine Fas ligand (CD95 Ligand). J. Interferon Cytokine Res. 21, 305-312 (2001). patient with autoimmune lymphoproliferative syndrome caused by a novel FASLG mutation. Pediatr. Res. 78, 603 (2015). 
Nicholson, D. W. et al. Identification and inhibition of the ICE/CED-3 protease necessary for mammalian apoptosis. Nature 376, 37 (1995).

69082 Dai, R., Frejtag, W., He, B., Zhang, Y. \& Mivechi, N. F. C-Jun NH2-terminal kinase targeting and phosphorylation of heat shock factor-1 suppress its transcriptional activity. J. Biol. Chem. 275, 18210-18218 (2000).

694

83 Cao, W.-X. et al. MLKL mediates apoptosis via a mutual regulation with PERK/elF2 $\alpha$ pathway in response to reactive oxygen species generation. Apoptosis 23, 521-531 (2018). Lu, X.-X. et al. PTEN inhibits cell proliferation, promotes cell apoptosis, and induces cell cycle arrest via downregulating the PI3K/AKT/hTERT pathway in lung adenocarcinoma A549 cells. BioMed research international 2016 (2016). Chen, C.-Y., Chen, J., He, L. \& Stiles, B. L. PTEN: tumor suppressor and metabolic regulator. Front. Endocrinol. (Lausanne) 9 (2018). Lee, Y. S. et al. Dysregulation of adipose glutathione peroxidase 3 in obesity contributes to local and systemic oxidative stress. Mol. Endocrinol. 22, 2176-2189 (2008). glutathione peroxidase (GPx-3) expression. J. Biol. Chem. 279, 26839-26845 (2004). Ighodaro, O.\& Akinloye, O. First line defence antioxidants-superoxide dismutase (SOD), catalase (CAT) and glutathione peroxidase (GPX): Their fundamental role in the entire antioxidant defence grid. Alexandria Journal of Medicine 54, 287-293 (2018). alkylhydroperoxide reductase (ahpC) mutation in Escherichia coli. Biochem. J. 307, 377-381 (1995).

90 Yamashita, H. et al. Characterization of human and murine PMP20 peroxisomal proteins that exhibit antioxidant activity in vitro. J. Biol. Chem. 274, 29897-29904 (1999).

91 Knoops, B. et al. Cloning and characterization of AOEB166, a novel mammalian antioxidant enzyme of the peroxiredoxin family. J. Biol. Chem. 274, 30451-30458 (1999).

92 Kang, S. W., Baines, I. C. \& Rhee, S. G. Characterization of a mammalian peroxiredoxin that contains one conserved cysteine. J. Biol. Chem. 273, 6303-6311 (1998).

93 Chen, J.-W., Dodia, C., Feinstein, S. I., Jain, M. K. \& Fisher, A. B. 1-Cys peroxiredoxin, a bifunctional enzyme with glutathione peroxidase and phospholipase A2 activities. J. Biol. Chem. 275, 28421-28427 (2000).

94 Schmieder, R. \& Edwards, R. Quality control and preprocessing of metagenomic datasets. Bioinformatics 27, 863-864 (2011).

95 Cunningham, F. et al. Ensembl 2015. Nucleic Acids Res. 43, D662-D669 (2014).

$96 \mathrm{Li}, \mathrm{B}$. \& Dewey, C. N. RSEM: accurate transcript quantification from RNA-Seq data with or without a reference genome. BMC Bioinformatics 12, 323 (2011).

97 Langmead, B. \& Salzberg, S. L. Fast gapped-read alignment with Bowtie 2. Nat. Methods 9, 357 (2012).

98 Robinson, M. D., McCarthy, D. J. \& Smyth, G. K. edgeR: a Bioconductor package for differential expression analysis of digital gene expression data. Bioinformatics 26, 139-140 (2010).

99 Reimand, J., Kull, M., Peterson, H., Hansen, J. \& Vilo, J. g: Profiler-a web-based toolset for functional profiling of gene lists from large-scale experiments. Nucleic Acids Res. 35, W193W200 (2007).

100 Reimand, J. et al. g: Profiler-a web server for functional interpretation of gene lists (2016 update). Nucleic Acids Res. 44, W83-W89 (2016).

101 Jassal, B. et al. The reactome pathway knowledgebase. Nucleic acids research 48, D498-D503, 
102 Krämer, A., Green, J., Pollard Jr, J. \& Tugendreich, S. Causal analysis approaches in ingenuity pathway analysis. Bioinformatics 30, 523-530 (2013).

103 Manjunath, S. et al. Genomic analysis of host - Peste des petits ruminants vaccine viral 46, 15, doi:10.1186/s13567-015-0153-8 (2015).

104 Szklarczyk, D. et al. STRING v11: protein-protein association networks with increased coverage, supporting functional discovery in genome-wide experimental datasets. Nucleic Acids Res. 47, D607-D613 (2018).

105 Shannon, P. et al. Cytoscape: a software environment for integrated models of biomolecular interaction networks. Genome Res. 13, 2498-2504 (2003).

106 Schmittgen, T. D. \& Livak, K. J. Analyzing real-time PCR data by the comparative C T method. Nat. Protoc. 3, 1101 (2008).

\section{Legends}

751 Figure 1: Overview of the work done : Two genetic groups (Tharparkar and Vrindavani) of 752 cattle were exposed to a temperature of $42{ }^{\circ} \mathrm{C}$ for 7 days. Heat stress indicators - Respiration rate

753 (RR), Rectal temperature and T3 level before exposure to heat (Oday - control group) and at $7^{\text {th }}$

754 day of exposure (treated) were measured to evaluate heat stress. At these time points, RNA was

755 isolated from PBMCs for high throughput sequencing. Transcriptome analysis was done to 756 identify differentially expressed genes (DEGs) under heat treatment in both genetic groups. 757 Genes involved in physiological processes (heat stress response, apoptosis, ubiquitination, 758 unfolded protein response and antioxidant level) that are commonly associated with heat stress 759 were compared between the two genetic groups. Further, functional annotation of DEGs was 760 done using IPA.

761 Figure 2: Respiration rate, Rectal Temperature and T3 level measured at 0 day (control) and 7 762 day post-heat exposure (treated) in Vrindavani and Tharparkar $(n=5)$ Levels sharing the same 763 superscript are not significantly $(\mathrm{P}>0.05)$ different from each other.

764 Figure 3: Expression of DEGs in Vrindavani and Tharparkar under heat stress: (a) Venn 765 diagrams showing unique/common DEGs between Vrindavani and Tharparkar (b) Number of 766 upregulated and downregulated in both genetic groups (c) Contrast in the expression of common 767 DEGs 
Figure 4: Canonical pathways activated/inactivated in (a) Vrindavani (b) Tharparkar under heat stress generated in the core analysis of Ingenuity pathway analysis tool. Orange color pathways are activated $(Z>2)$ and blue color pathways are inactivated $(Z<-2)$. Height of the bar graphs indicates - $\log (\mathrm{p}$-value) and line graph showing the ratio of list genes found in each pathway over

772 the total number of genes in that pathway.

773 Figure 5: Canonical pathways generated in Ingenuity Pathway Analysis of Oncostatin M

774 signaling pathway of DEGs in (A) Vrindavani, (B) Tharparkar. Genes that were upregulated are

775 shown in red and downregulated in green. The intensity of red and green corresponds to an 776 increase and decrease, respectively, in $\log 2$ fold change. Genes in grey were not significantly

777 dysregulated and those in white are not present in the dataset but have been incorporated in the 778 network through the relationship with other molecules by IPA.

779 Figure 6: Canonical pathways generated in Ingenuity Pathway Analysis of PTEN signaling 780 pathway of DEGs in (A) Vrindavani, (B) Tharparkar. Genes that were upregulated are shown in 781 red and downregulated in green. The intensity of red and green corresponds to an increase and 782 decrease, respectively, in Log2 fold change. Genes in grey were not significantly dysregulated 783 and those in white are not present in the dataset but have been incorporated in the network 784 through the relationship with other molecules by IPA.

785 Figure 7: Contrast in the expression of genes involved in heat stress response, apoptosis, 786 ubiquitination, unfolded protein response and balance in the production of ROS and antioxidants 787 between two genetic groups.

788 Figure 8: Predicted interplay of molecules that is underway during heat stress in both 789 groups : Heat stress causes unfolding of native proteins. HSP70 acts as a chaperone to facilitate 790 refolding to restore the structure of unfolded proteins. Under normal condition, HSP70 is bound 791 to HSF1 thereby preventing HSF1 to promote transcription of HSP70. Under heat stress ATP 792 binds to the HSP70 and HSF1 complex to release HSF1, promoting the binding of the unfolded 793 protein to HSP70 and ATP. CAMK2D that induces the transcription of HSP70 via HSF1 was 794 found downregulated in Tharparkar. GSK3A that inhibits the trimerization of HSF1 that is 795 needed for the induction of HSP70 expression was found upregulated in Tharparkar. The 796 decreased level of HSP70 in Vrindavani makes it inevitable that such negative feedbacks would 797 further reduce its level and GSK3A was found downregulated and CAMK2D, upregulated. 
798 Further, in Tharparkar, HSP70 tends to activate ubiquitination pathway to decrease the

799 accumulation of unfolded proteins that can't be refolded by it. This pathway activation is

800 supported by upregulation of E3 ligases (UBE2G1, UBE2S, and UBE2H) in Tharparkar.

801 However, the E3 ligase in Vrindavani was found downregulated. With HSP70 being upregulated

802 and having cytoprotection activity, Tharparkar shows the decline in apoptosis as compared to

803 Vrindavani. This is supported by downregulation of BCL2L11, FASLG, MLKL, CASP3,

804 MAPK8 and VIM in Tharparkar and vice-versa. Besides, higher expression of the antioxidants

805 (SOD, CAT, GPX) in Tharparkar enables it to cope up with higher levels of free radicals

806 generated as a result of heat stress while Vrindavani is unable to do so. Green arrow indicates

807 downregulation and Maroon arrow indicates upregulation.

808 Supplementary.Figure 1: Comparison of activated/inactivated pathways in Vrindavani and

809 Tharparkar. Activated pathways have Z score $>2$ and indicated by red colour while inactivated

810 pathways are having $\mathrm{Z}$ score $<-2$ and indicated by green colour.

811 Supplementary Figure 2: Comparison of activated/inactivated miRNAs in Vrindavani and

812 Tharparkar as predicted by IPA upstream analysis. Activated pathways have Z score $>2$ and

813 indicated by red colour while inactivated pathways are having $\mathrm{Z}$ score $<-2$ and indicated by

814 green colour.

815 Supplementary Figure 3: Comparison of activated/inactivated Transcription factors as 816 predicted by IPA upstream analysis (Transcription factors of Vrindavani are red-coloured and

817 Tharparkar are blue-coloured ) vis-à-vis their Log2FC in both genetic groups. The encircled ones 818 are common to both groups.

819 Supplementary Figure 4: Predicted Protein-protein interaction network of expressed genes 820 common to Tharparkar and Vrindavani. The diameter of the node represents the 821 connectivity/degree of the node among the genes.

822 Supplementary Figure 5: Validation of RNA sequencing data by Real-Time data in Vrindavani 823 (a) and Tharparkar (b). The expression of 10 selected genes was found in concordance with RNA 824 Sequencing data. The correlation ( $\mathrm{r} 2=0.9942$ in (a) and 0.9972 in (b) ) was found to be 825 significant $(\mathrm{P}<.01)$ in both cases. 
bioRxiv preprint doi: https://doi.org/10.1101/2020.04.09.031153; this version posted January 1, 2021. The copyright holder for this preprint (which was not certified by peer review) is the author/funder. All rights reserved. No reuse allowed without permission.

826 

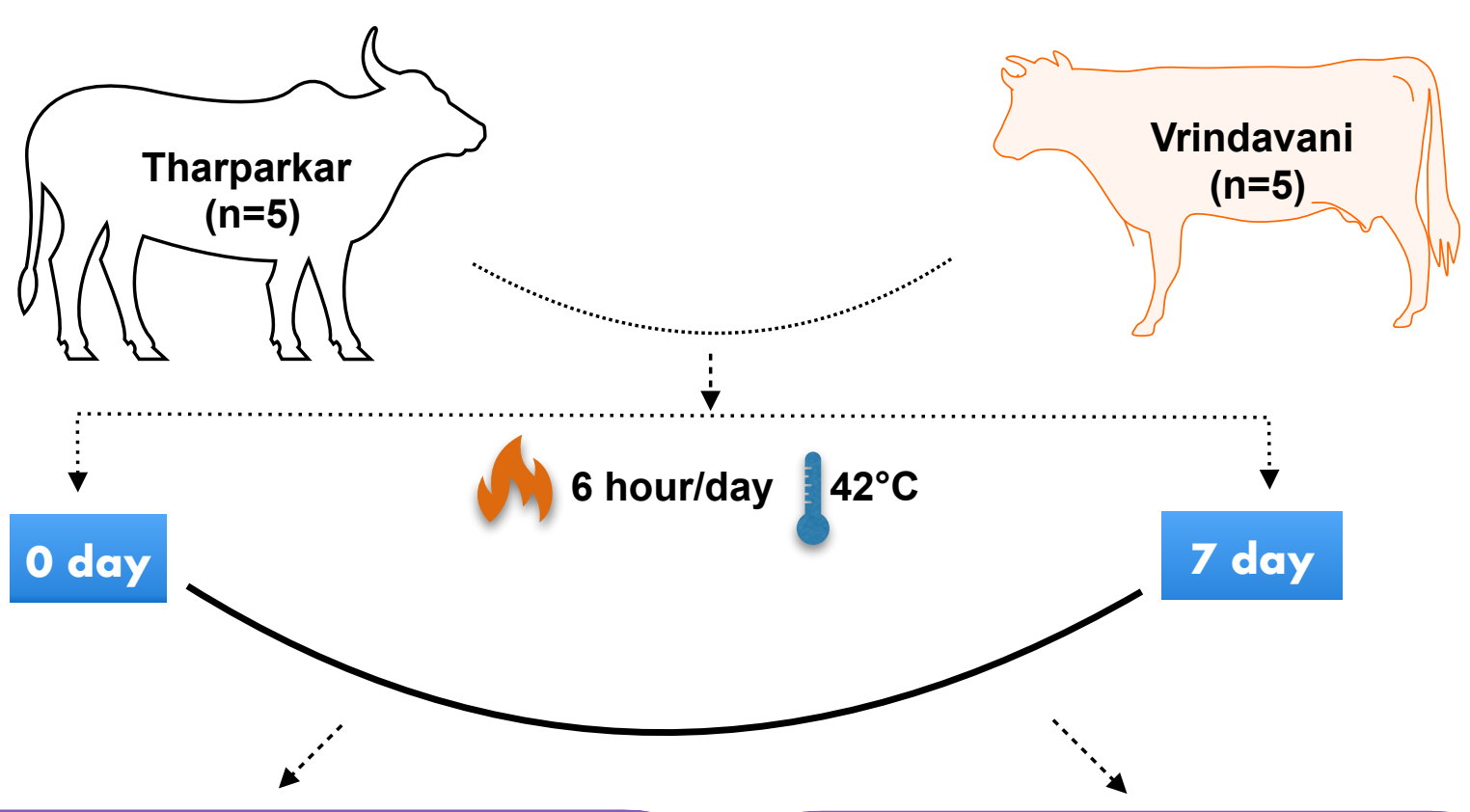

PBMC isolation and RNA sequencing

Physiological Parameters

\section{$\downarrow$}

Identification of differentially expressed genes

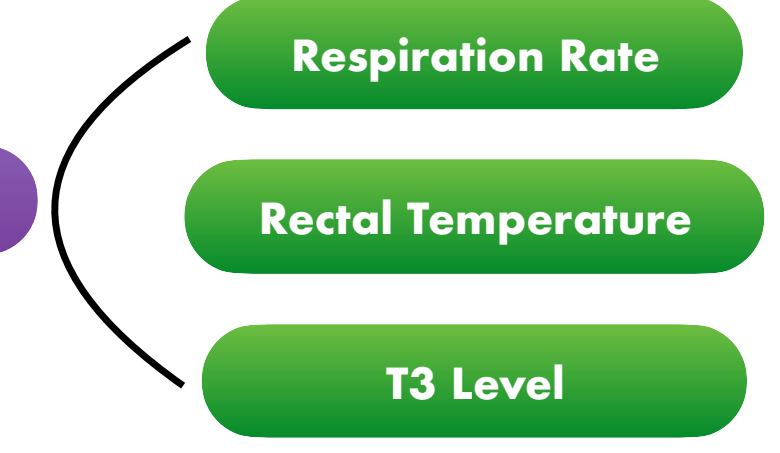

$\downarrow$

Functional analysis of differentially expressed genes of both genetic groups, common genes and unique genes

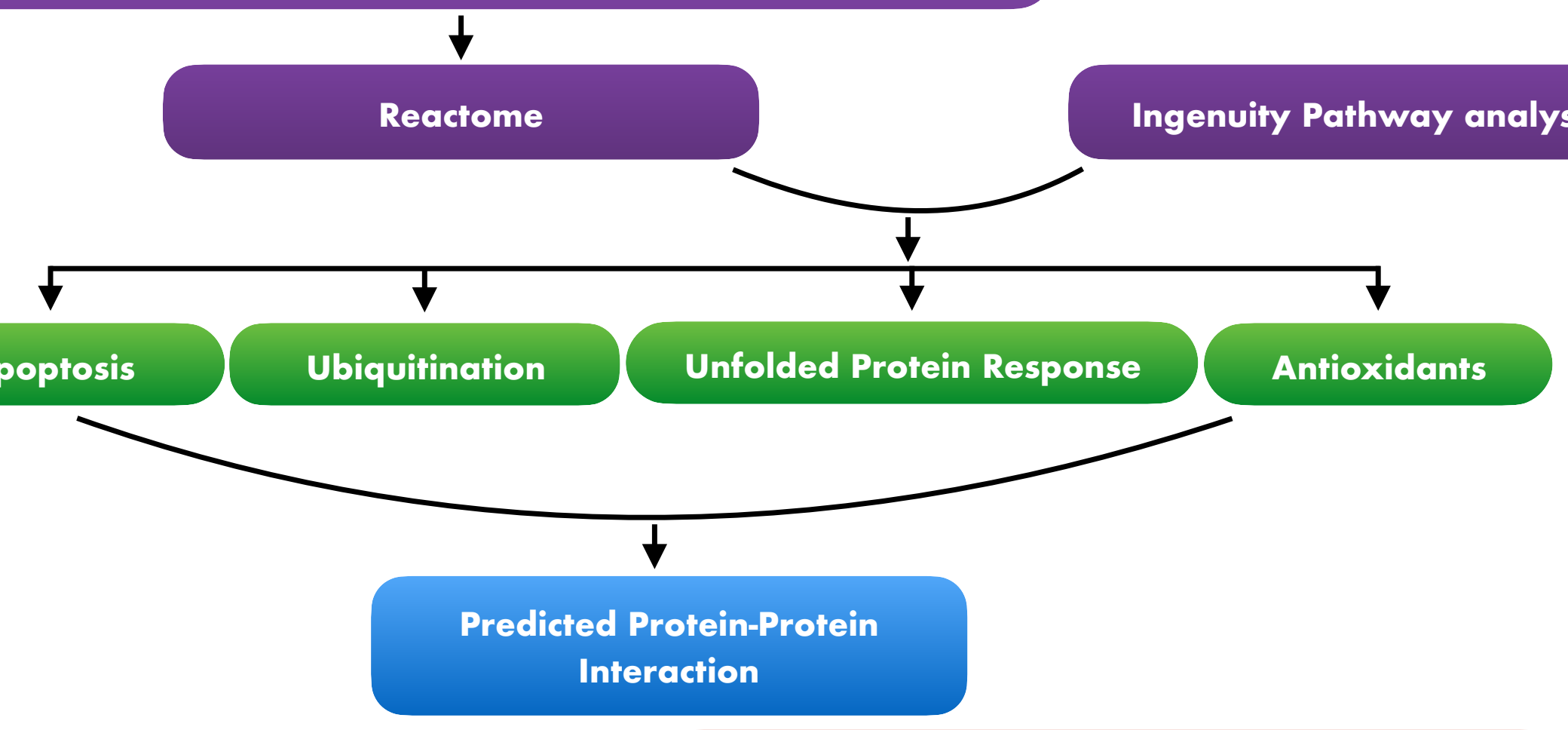

Identification of activated/ inactivated signalling pathways

Prediction of microRNAs and Transcription Factors

Diseases and Functions 


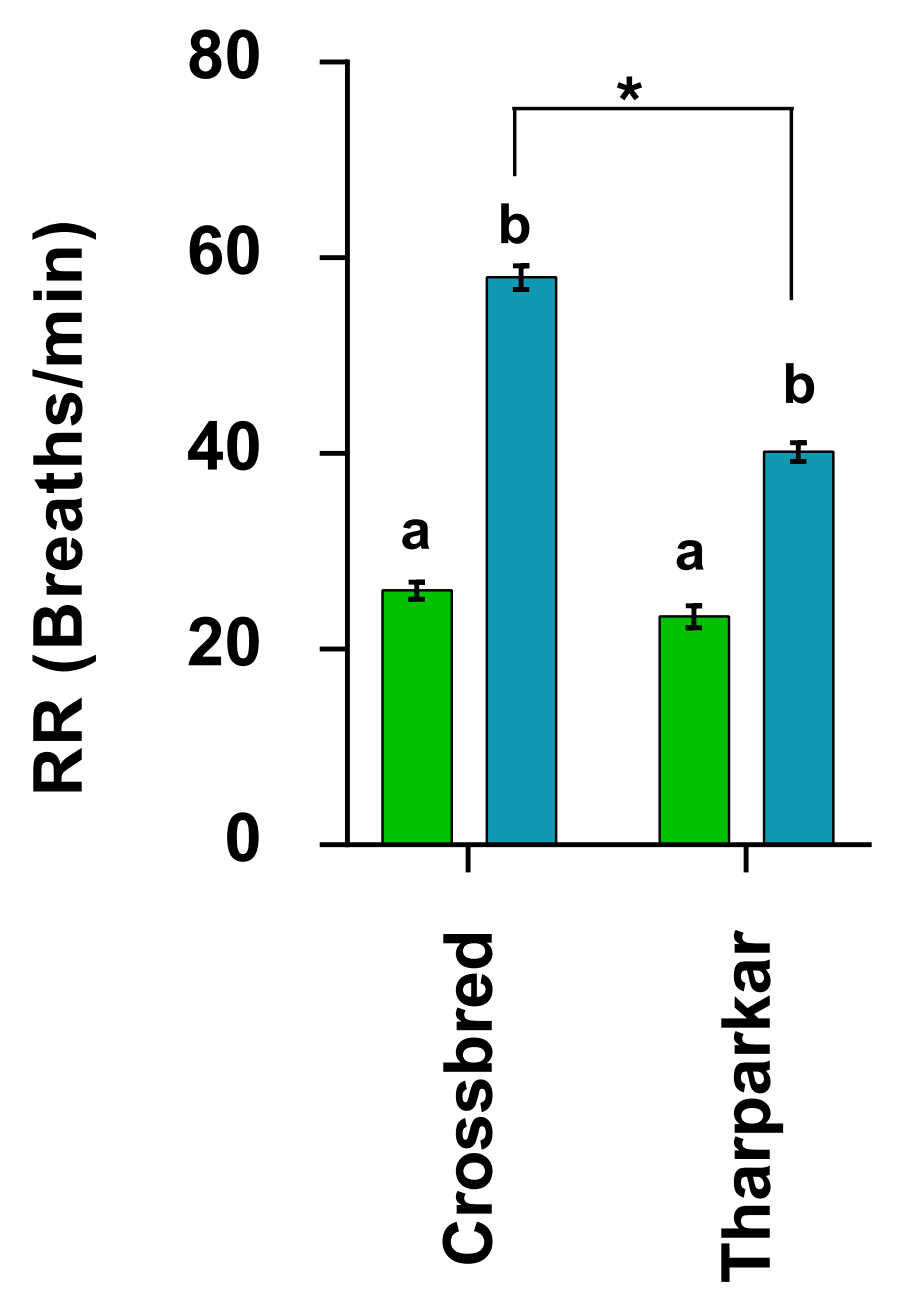

(a)
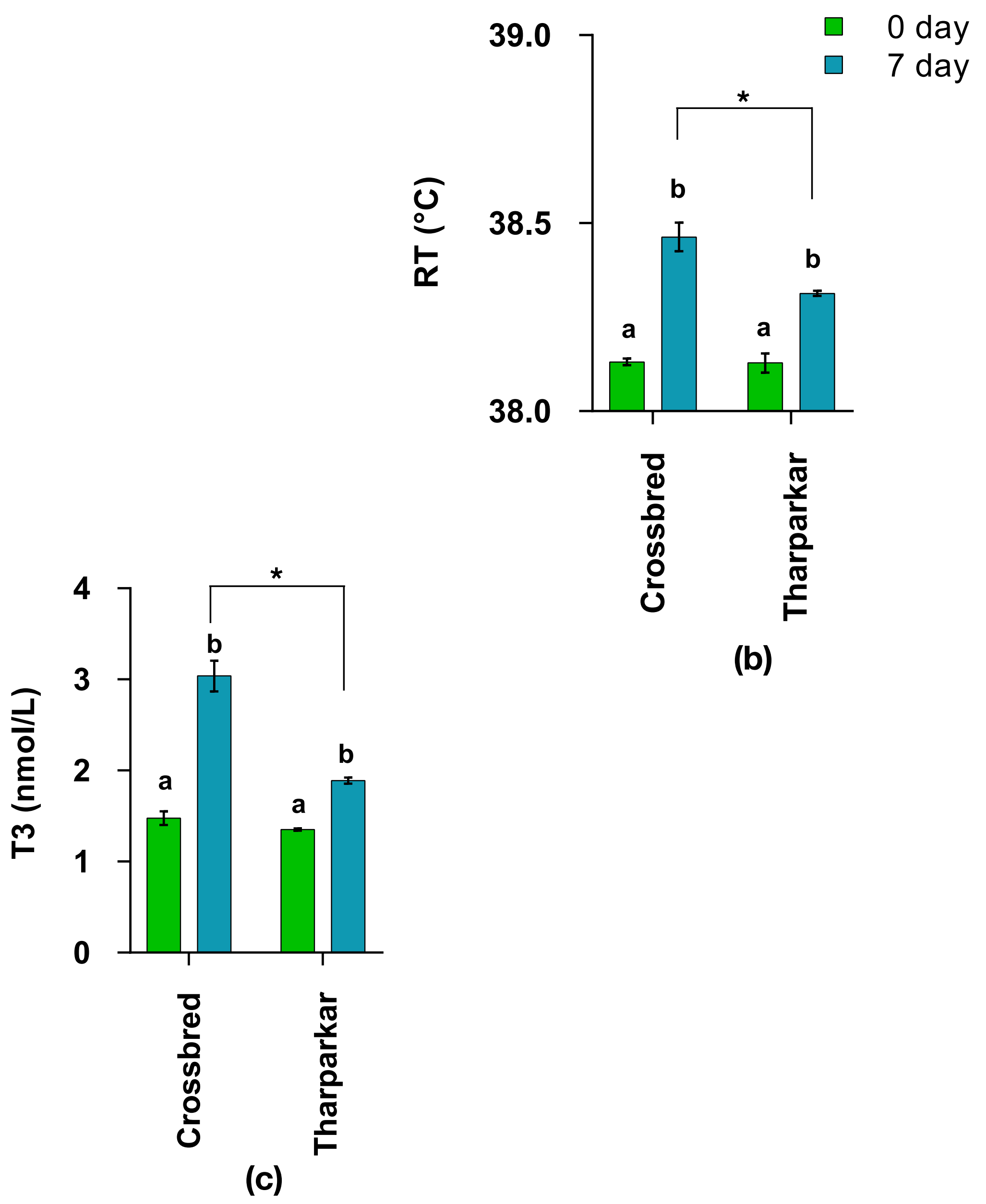


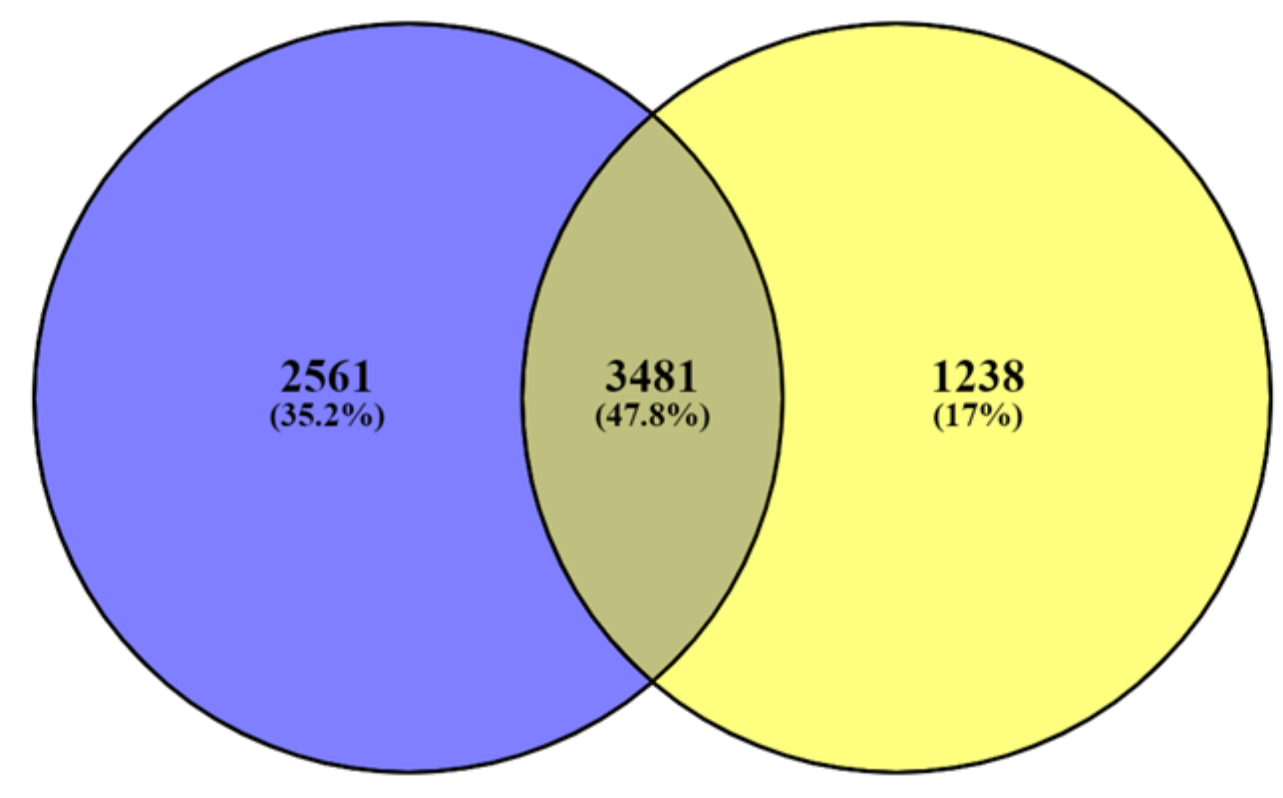

\section{Down-regulated \\ Up-regulated}

\section{Crossbred_DEGs}

Tharparkar_DEGs
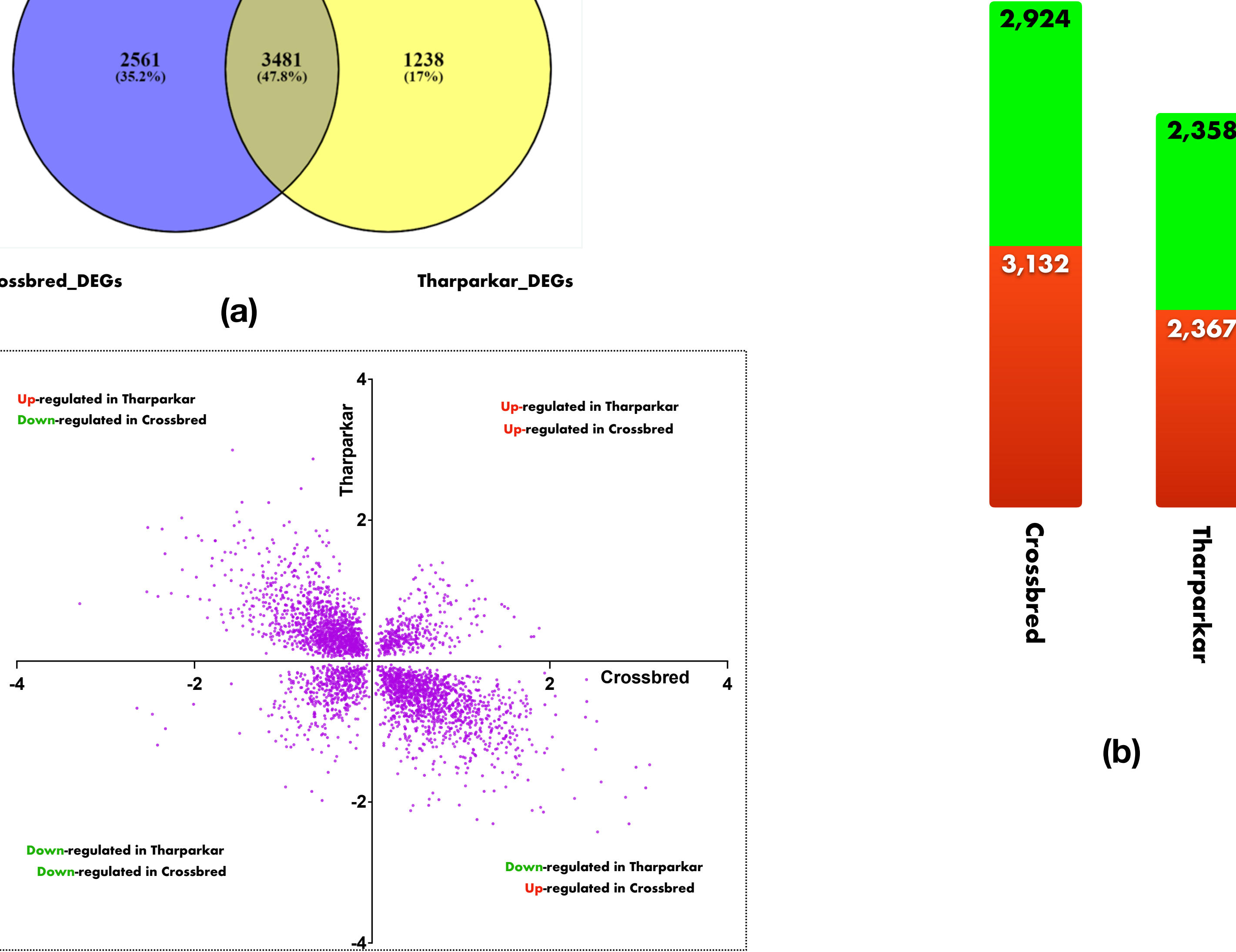

Up-regulated in Tharparkar

Up-regulated in Crossbred

Up-regulated in Tharparkar

Down-regulated in Crossbred

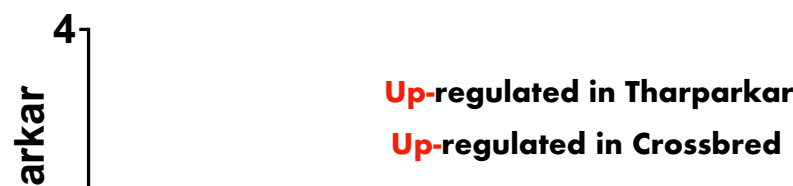

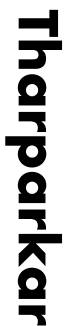

(c) 
$\square$ positive z-score $\square$ z-score $=0 \quad \square$ negative z-score $\square$ no activity pattern available $-\square-\log (\mathrm{p}$-value)

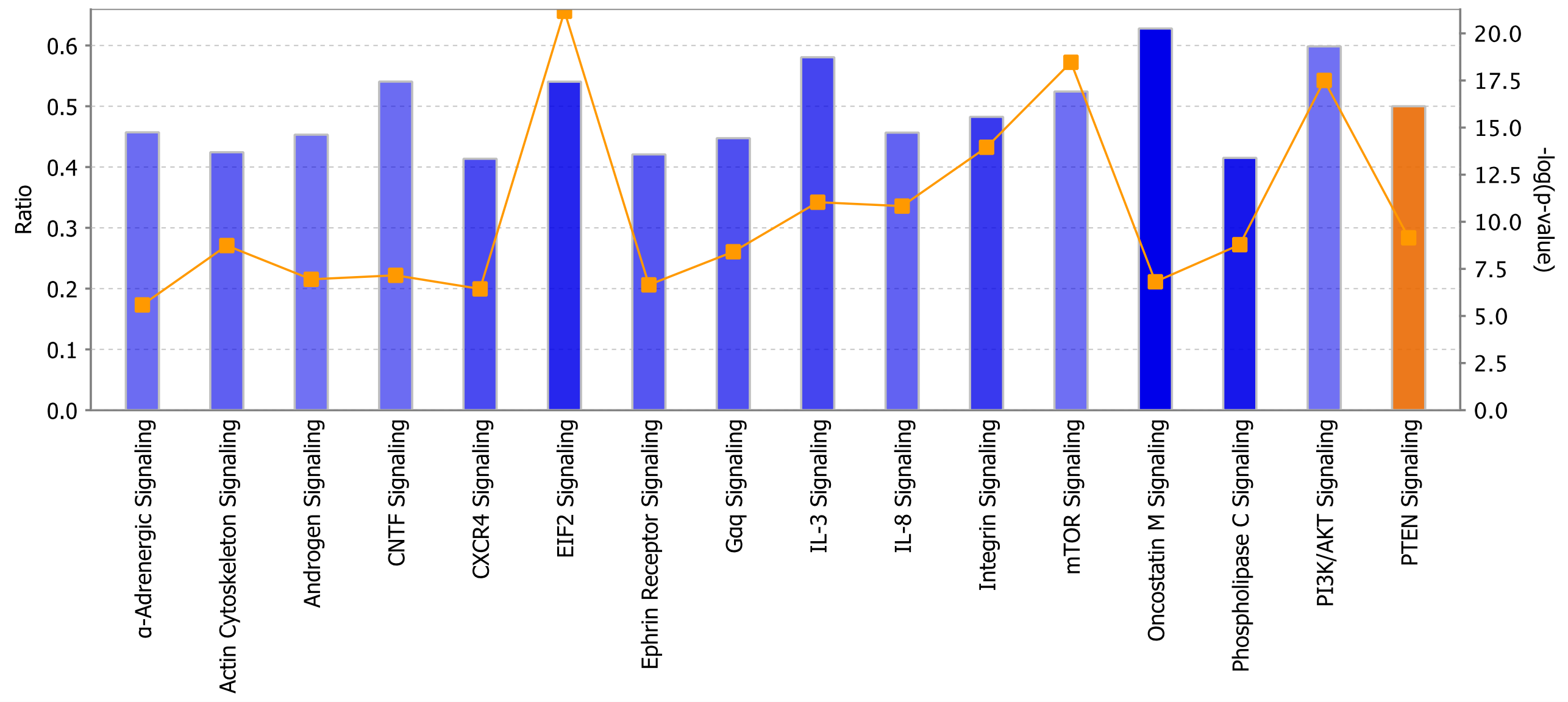

(a)

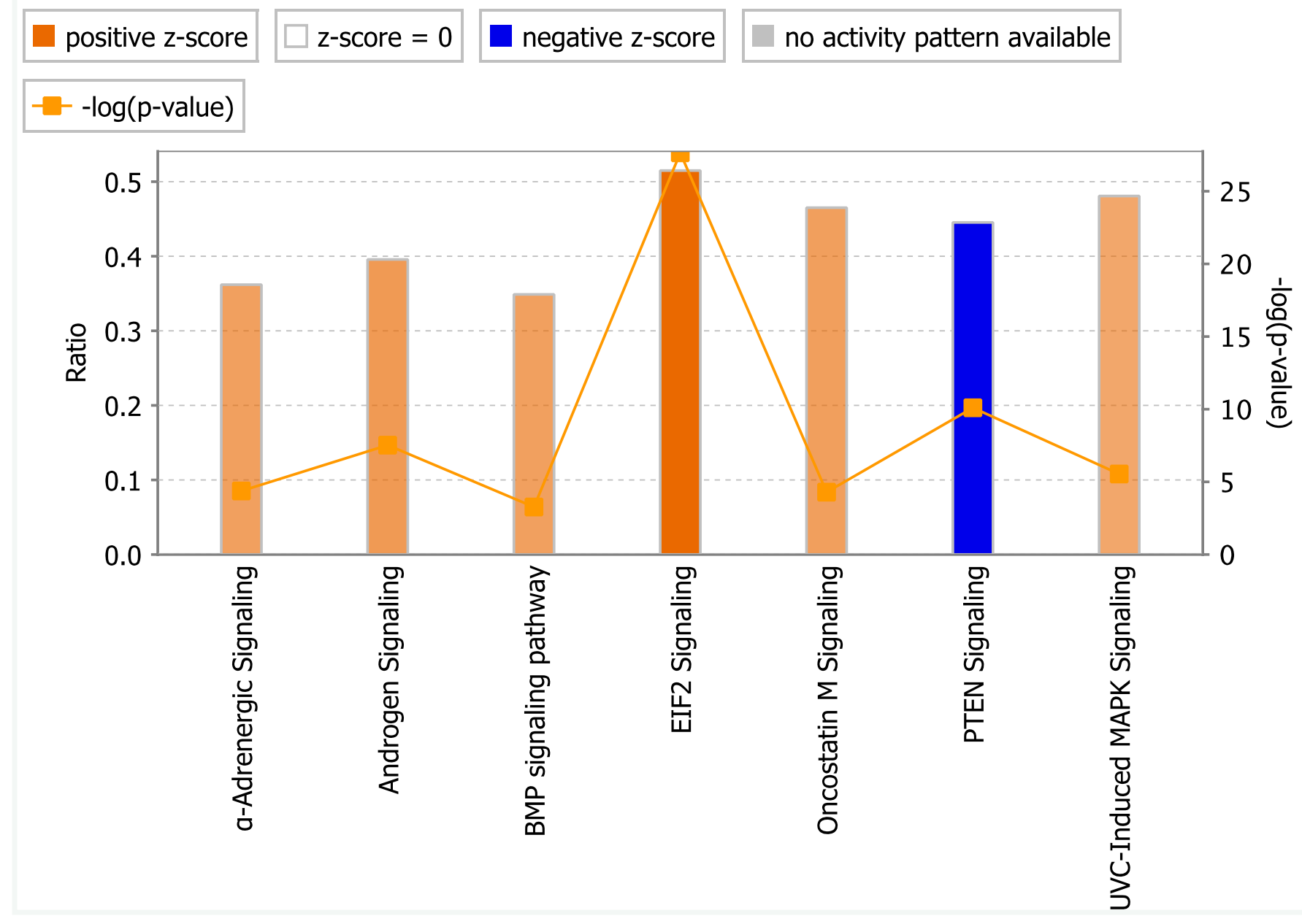

(b) 
Extracellular space

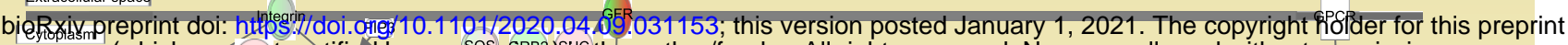

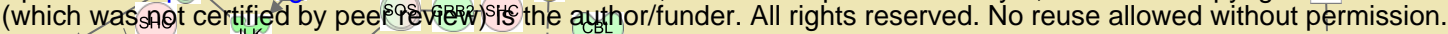

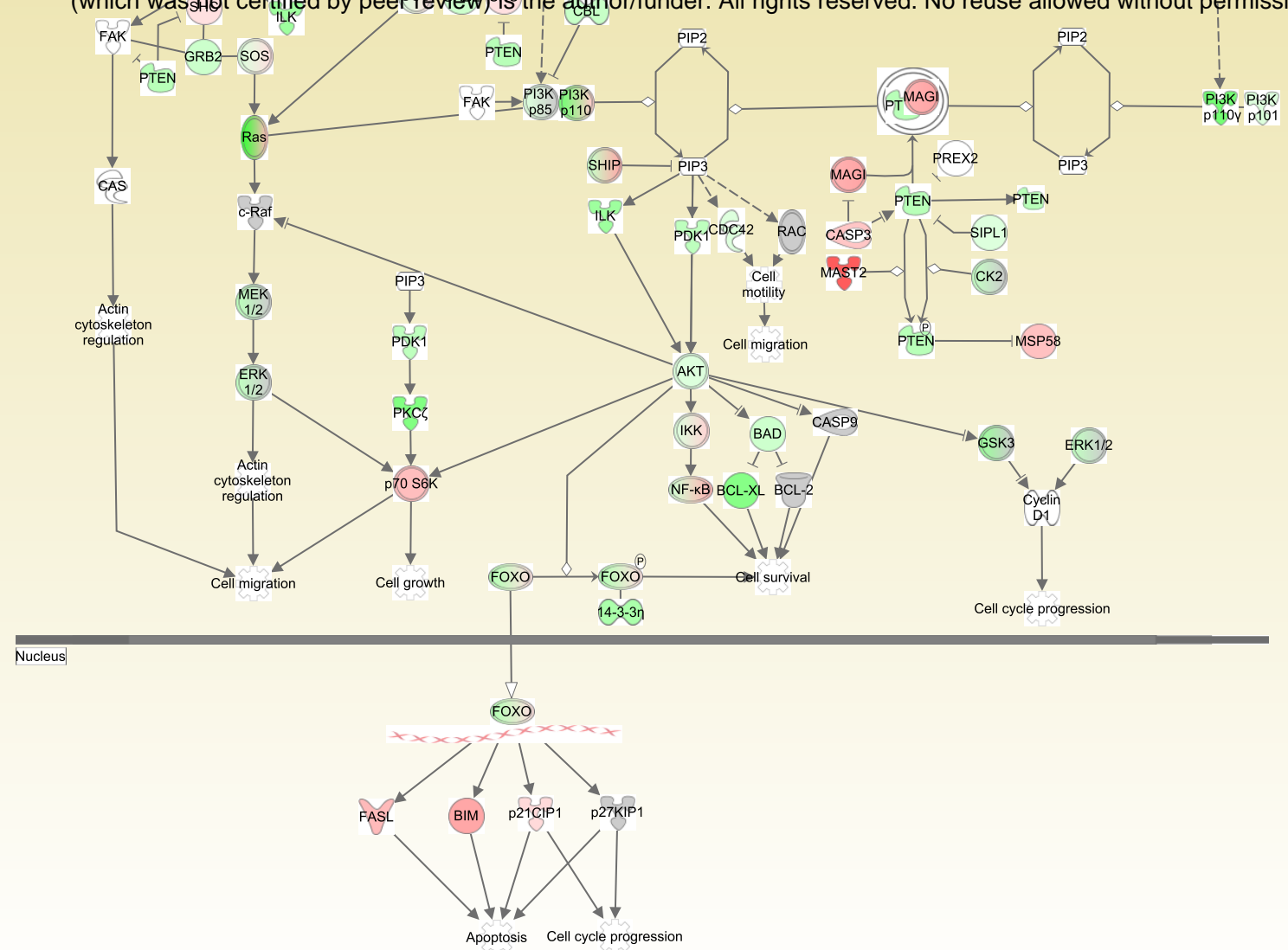

(a)

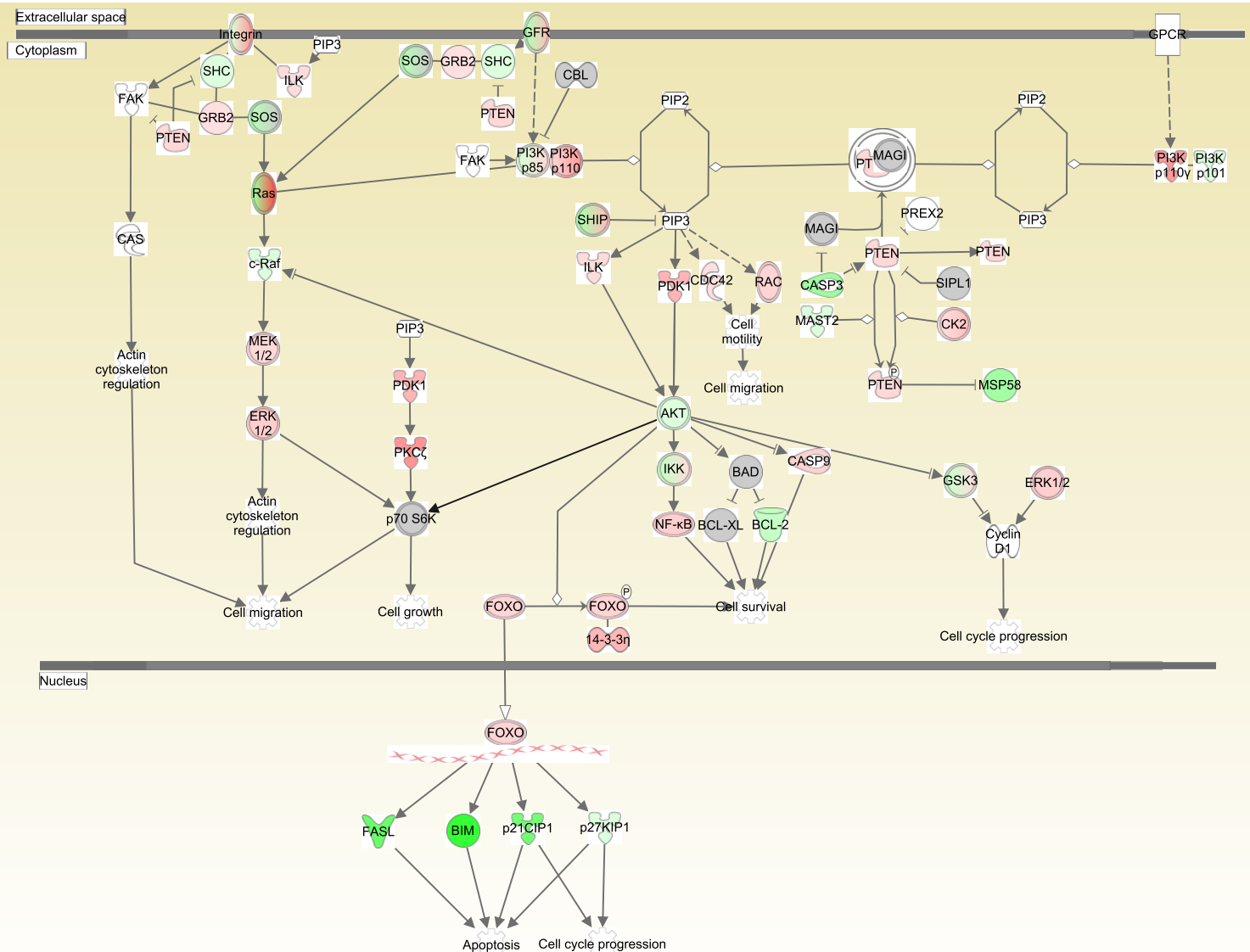




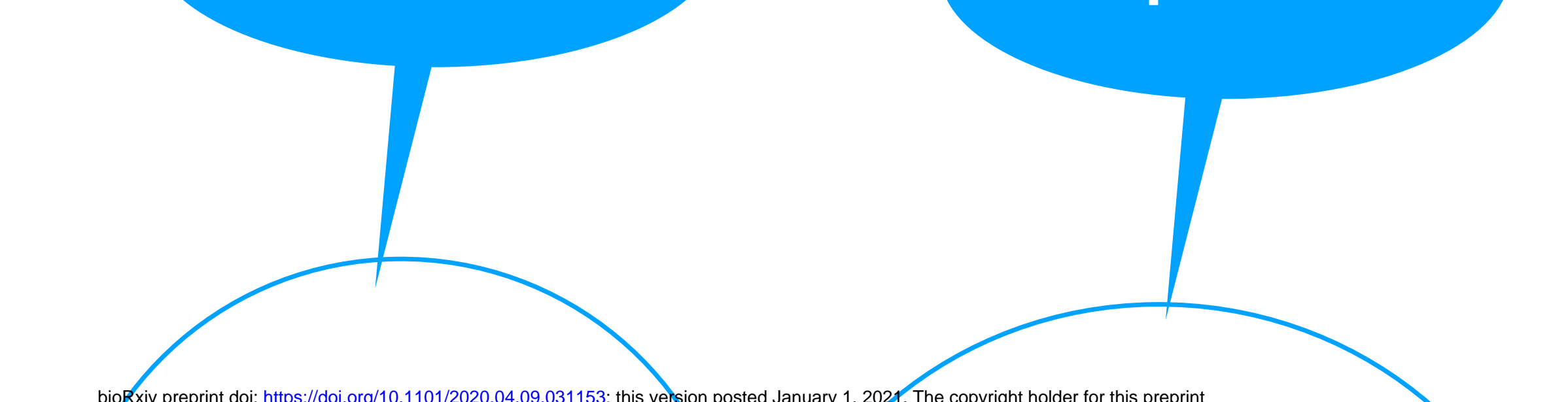

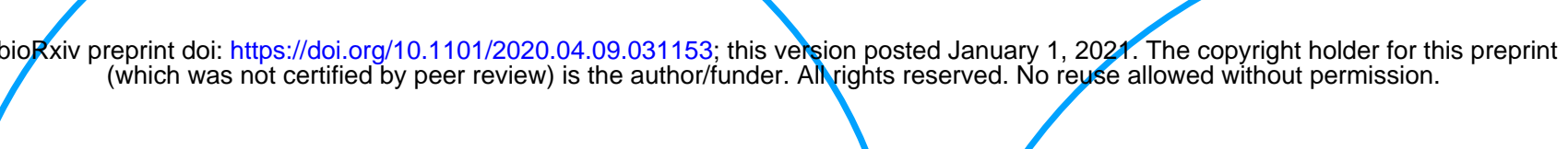

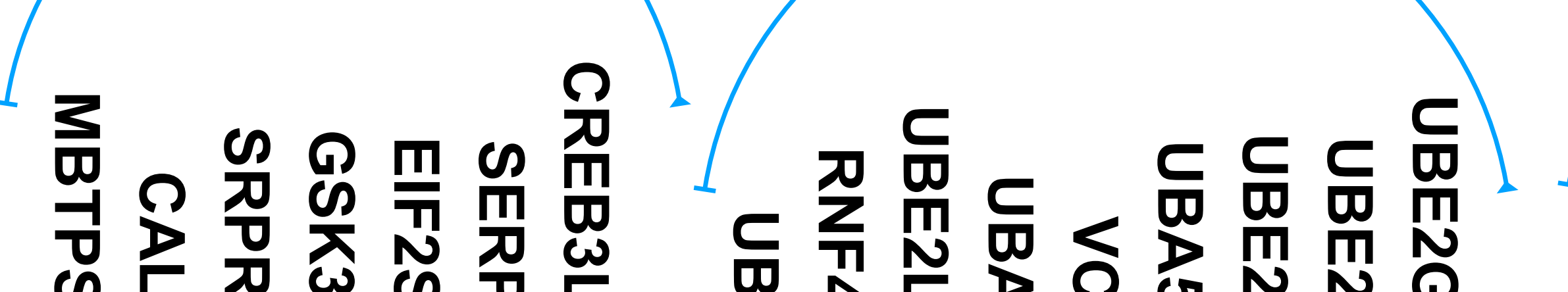

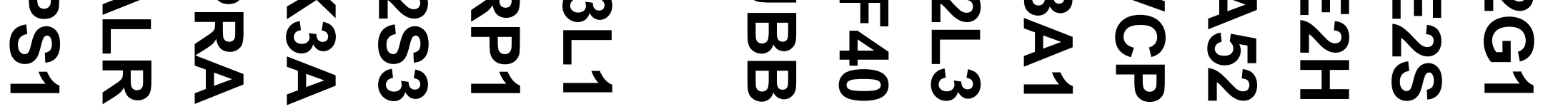

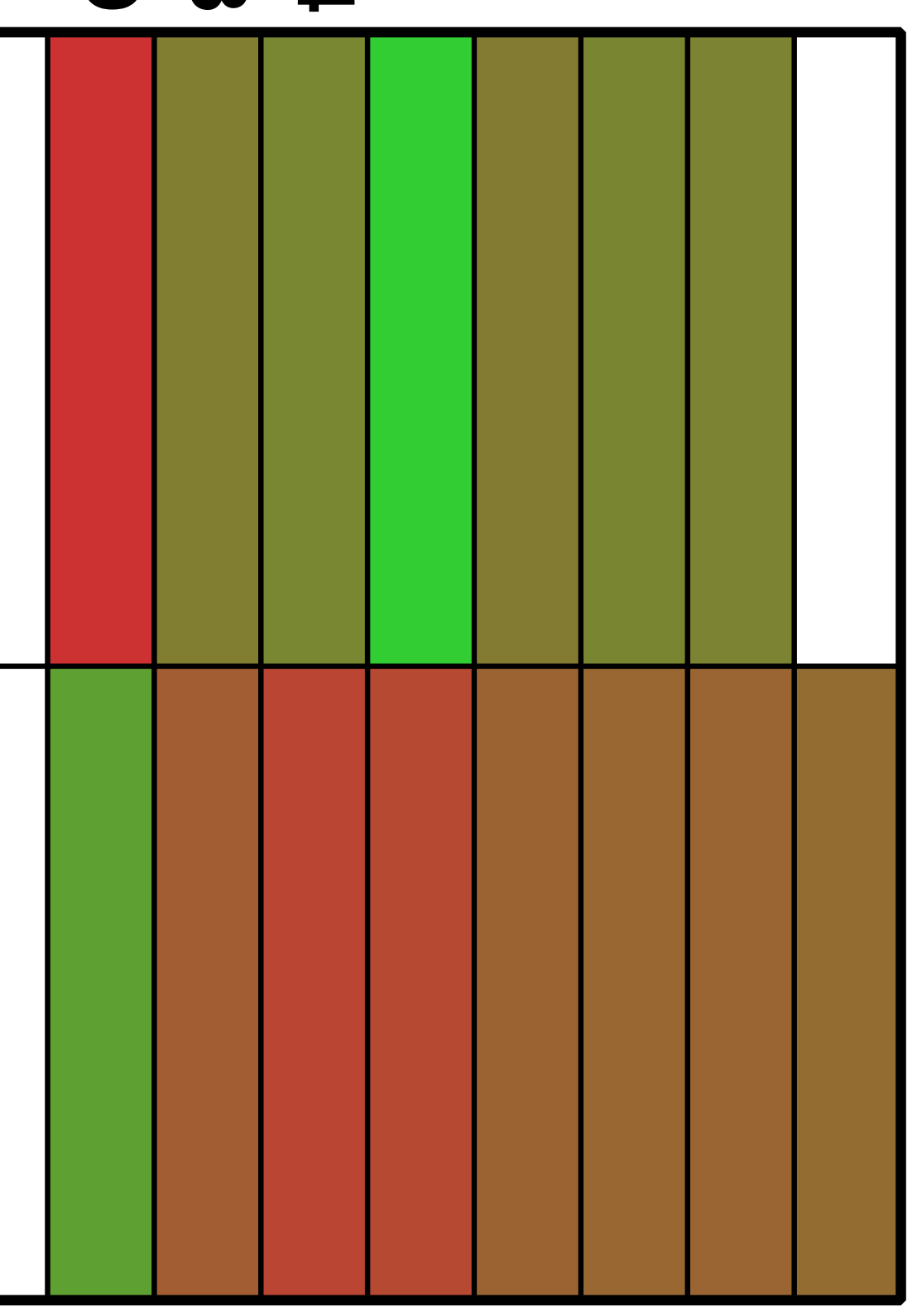

$\rightarrow$ 


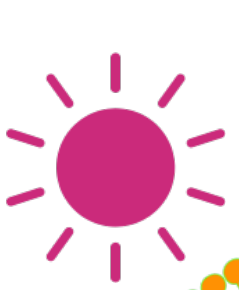

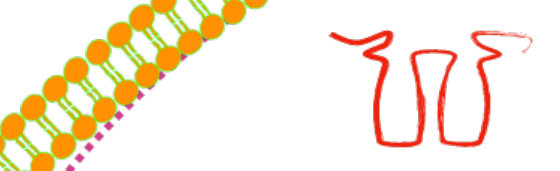

Heat stress causes unfolding of native proteins

HSF1

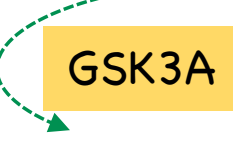

HSF1

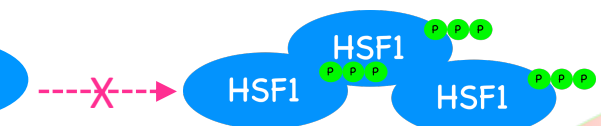

GSK3A, HSF1

Tharparkar

Crossbred

1

HSF1

HSF1

eo

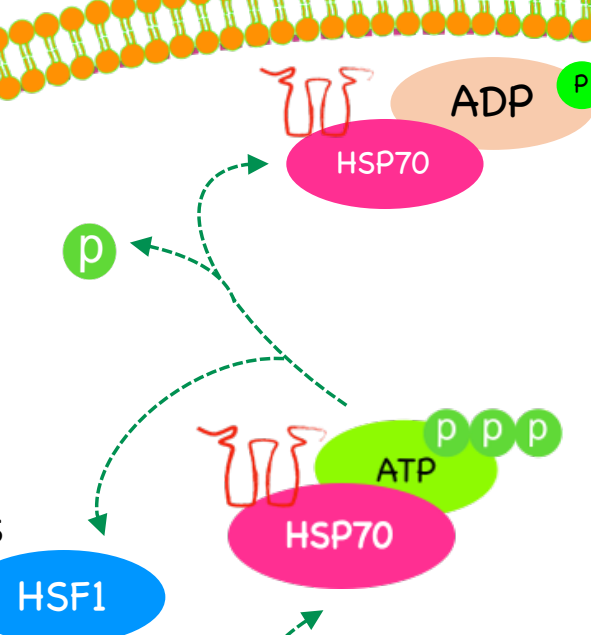

HSF1

Tharparkar
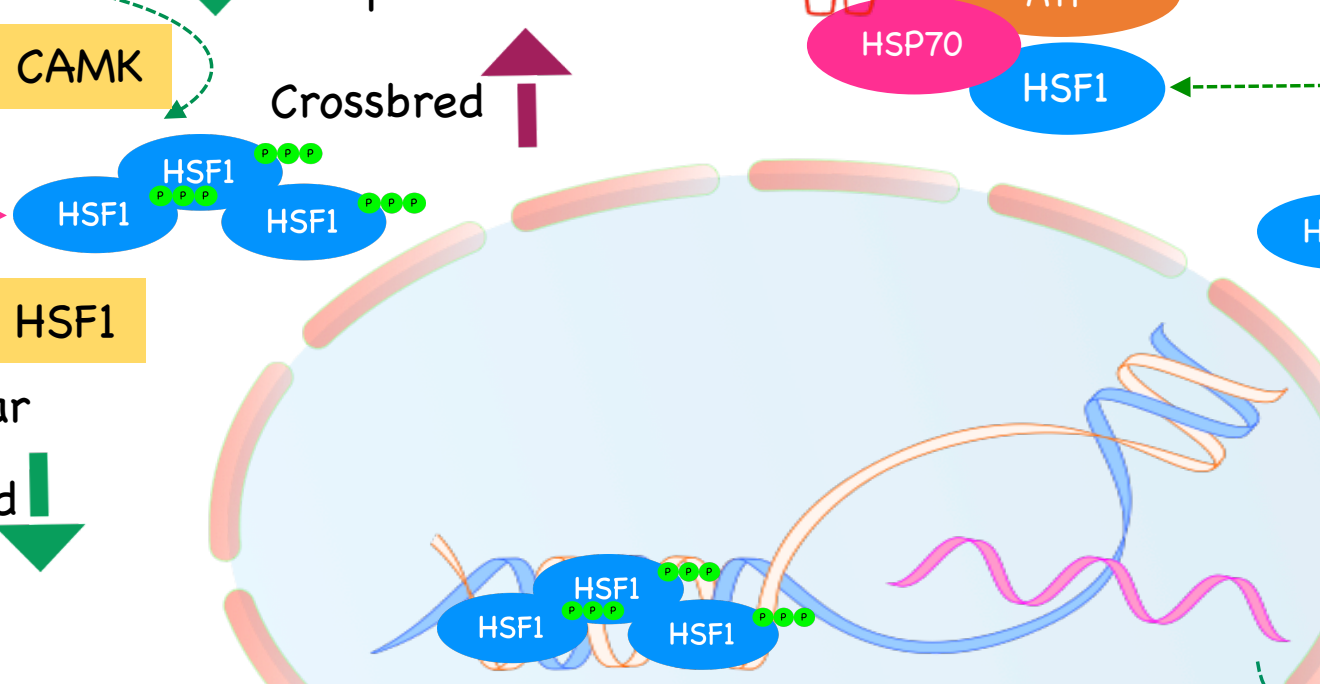

HSE

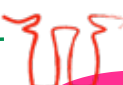

ATP P P P

HSF1
Tharparkar

Crossbred

UBE2G1, UBE2S, UBE2H

UBE2G1 UBE2S, UBE2H

Ubiquitination pathway

HSF1

HSF1

HSPAIA HSPA8

HSF1

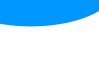

HSP70 HSP70

HSP70

Tharparkar

Crosisbred

BCL2L11, FASLG, MLKL, CASP3, MAPK8 and VIM Tharparkar

Crossbred
SOD, CAT, GPX

Heat stress causes ROS Production

Tharparkar Crossbred 